\title{
The strong simple connectedness of tame algebras with separating almost cyclic coherent Auslander-Reiten components
}

\author{
Piotr Malicki ${ }^{1}$
}

Received: 20 December 2019 / Accepted: 21 December 2020 / Published online: 30 January 2021

(C) The Author(s) 2021

\begin{abstract}
We study the strong simple connectedness of finite-dimensional tame algebras over an algebraically closed field, for which the Auslander-Reiten quiver admits a separating family of almost cyclic coherent components. As the main application we describe all analytically rigid algebras in this class.
\end{abstract}

Keywords Strongly simply connected algebra · Auslander-Reiten quiver · Generalized multicoil

Mathematics Subject Classification (2010) Primary 16G70 · Secondary 16G20

\section{Introduction and the main results}

Throughout the paper $k$ will denote a fixed algebraically closed field. By an algebra is meant an associative finite-dimensional $k$-algebra with an identity, which we shall assume (without loss of generality) to be basic. Then such an algebra has a presentation $A \cong k Q_{A} / I$, where $Q_{A}=\left(Q_{0}, Q_{1}\right)$ is the ordinary quiver of $A$ with the set of vertices $Q_{0}$ and the set of arrows $Q_{1}$ and $I$ is an admissible ideal in the path algebra $k Q_{A}$ of $Q_{A}$. If the quiver $Q_{A}$ has no oriented cycles, the algebra $A$ is said to be triangular. For an algebra $A$, we denote by $\bmod A$ the category of finitely generated right $A$-modules, and by ind $A$ a full subcategory of $\bmod A$ consisting of a complete set of representatives of the isomorphism classes of indecomposable modules. We shall denote by $\operatorname{rad}_{A}$ the Jacobson radical of $\bmod A$, and by $\operatorname{rad}_{A}^{\infty}$ the intersection of all powers $\operatorname{rad}_{A}^{i}, i \geq 1$, of $\operatorname{rad}_{A}$. Moreover, we denote by $\Gamma_{A}$ the Auslander-Reiten quiver of $A$, and by $\tau_{A}$ and $\tau_{A}^{-}$the Auslander-Reiten translations $D \mathrm{Tr}$

\section{Presented by: Christof Geiss}

Dedicated to Ibrahim Assem on the occasion of his 70th birthday.

Piotr Malicki

pmalicki@mat.umk.pl

1 Faculty of Mathematics and Computer Science, Nicolaus Copernicus University,

Chopina 12/18, 87-100, Toruń, Poland 
and $\operatorname{Tr} D$, respectively. We will not distinguish between a module in ind $A$ and the vertex of $\Gamma_{A}$ corresponding to it. Following [39] a family $\mathcal{C}$ of components is said to be generalized standard if $\operatorname{rad}_{A}^{\infty}(X, Y)=0$ for all modules $X$ and $Y$ in $\mathcal{C}$. We note that different components in a generalized standard family $\mathcal{C}$ are orthogonal, and all but finitely many $\tau_{A}$-orbits in $\mathcal{C}$ are $\tau_{A}$-periodic (see [39, (2.3)]). We refer to [31] for the structure and homological properties of arbitrary generalized standard Auslander-Reiten components of algebras.

Following Assem and Skowroński [8] a triangular algebra $A$ is called simply connected if, for any presentation $A \cong k Q_{A} / I$ of $A$ as a bound quiver algebra, the fundamental group $\pi_{1}\left(Q_{A}, I\right)$ of $\left(Q_{A}, I\right)$ is trivial (see Section 2). The importance of these algebras follows from the fact that often we may reduce (using techniques of Galois coverings) study of the module category of an algebra to that for the corresponding simply connected algebras. Recall that $A$ is called strongly simply connected [38] if every convex subcategory of $A$ is simply connected.

From Drozd's Tame and Wild Theorem [16] the class of algebras may be divided into two disjoint classes. One class consists of the tame algebras for which the indecomposable modules occur, in each dimension $d$, in a finite number of discrete and a finite number of one-parameter families. The second class is formed by the wild algebras whose representation theory comprises the representation theories of all finite dimensional algebras over $k$. Hence, a classification of the finite dimensional modules is only feasible for tame algebras. It has been shown by Crawley-Boevey [15] that, if $A$ is a tame algebra, then, for any dimension $d \geq 1$, all but finitely many isomorphism classes of indecomposable $A$-modules of dimension $d$ are invariant on the action of $\tau_{A}$, and hence, by a result due to Hoshino [19], lie in stable tubes of rank one in $\Gamma_{A}$. The indecomposable modules over tame algebras which do not lie in stable tubes of rank one are called discrete.

A prominent role in the representation theory of algebras is played by the algebras with separating families of Auslander-Reiten components. A concept of a separating family of tubes has been introduced by Ringel in $[33,34]$ who proved that they occur in the Auslander-Reiten quivers of hereditary algebras of Euclidean type, tubular algebras, and canonical algebras. In order to deal with wider classes of algebras, the following more general concept of a separating family of Auslander-Reiten components was proposed by Assem, Skowroński and Tomé in [11] (see also [27]). A family $\mathcal{C}=\left(\mathcal{C}_{i}\right)_{i \in I}$ of components of the Auslander-Reiten quiver $\Gamma_{A}$ of an algebra $A$ is called separating in $\bmod A$ if the components of $\Gamma_{A}$ split into three disjoint families $\mathcal{P}^{A}, \mathcal{C}^{A}=\mathcal{C}$ and $\mathcal{Q}^{A}$ such that:

(S1) $\mathcal{C}^{A}$ is a sincere generalized standard family of components;

(S2) $\operatorname{Hom}_{A}\left(\mathcal{Q}^{A}, \mathcal{P}^{A}\right)=0, \operatorname{Hom}_{A}\left(\mathcal{Q}^{A}, \mathcal{C}^{A}\right)=0, \operatorname{Hom}_{A}\left(\mathcal{C}^{A}, \mathcal{P}^{A}\right)=0$;

(S3) any homomorphism from $\mathcal{P}^{A}$ to $\mathcal{Q}^{A}$ in $\bmod A$ factors through the additive category $\operatorname{add}\left(\mathcal{C}^{A}\right)$ of $\mathcal{C}^{A}$.

Then we say that $\mathcal{C}^{A}$ separates $\mathcal{P}^{A}$ from $\mathcal{Q}^{A}$ and write $\Gamma_{A}=\mathcal{P}^{A} \cup \mathcal{C}^{A} \cup \mathcal{Q}^{A}$. We note that then $\mathcal{P}^{A}$ and $\mathcal{Q}^{A}$ are uniquely determined by $\mathcal{C}^{A}$ (see [11, (2.1)] or [34, (3.1)]). Moreover, $\mathcal{C}^{A}$ is called sincere if any simple $A$-module occurs as a composition factor of a module in $\mathcal{C}^{A}$. We note that if $A$ is an algebra of finite representation type that $\mathcal{C}^{A}=\Gamma_{A}$ is trivially a unique separating component of $\Gamma_{A}$, with $\mathcal{P}^{A}$ and $\mathcal{Q}^{A}$ being empty. Frequently, we may recover $A$ completely from the shape and categorical behavior of the separating family $\mathcal{C}_{A}$ of components of $\Gamma_{A}$. For example, the tilted algebras [20,34], or more generally double tilted algebras [32], are determined by their (separating) connecting components. Further, it was proved in [22] that the class of algebras with a separating family of stable tubes coincides 
with the class of concealed canonical algebras. This was extended in [23] to a characterization of all quasitilted algebras of canonical type, for which the Auslander-Reiten quiver admits a separating family of semiregular tubes. Then, the latter has been extended in [27] to a characterization of algebras with a separating family of almost cyclic coherent AuslanderReiten components. Recall that a component $\Gamma$ of an Auslander-Reiten quiver $\Gamma_{A}$ is called almost cyclic if all but finitely many modules in $\Gamma$ lie on oriented cycles contained entirely in $\Gamma$. Moreover, a component $\Gamma$ of $\Gamma_{A}$ is said to be coherent if the following two conditions are satisfied:

(C1) For each projective module $P$ in $\Gamma$ there is an infinite sectional path $P=X_{1} \rightarrow X_{2} \rightarrow \cdots \rightarrow X_{i} \rightarrow X_{i+1} \rightarrow X_{i+2} \rightarrow \cdots$ in $\Gamma$

(C2) For each injective module $I$ in $\Gamma$ there is an infinite sectional path $\cdots \rightarrow Y_{j+2} \rightarrow Y_{j+1} \rightarrow Y_{j} \rightarrow \cdots \rightarrow Y_{2} \rightarrow Y_{1}=I$ in $\Gamma$.

It has been proved in [27, Theorem A] that the Auslander-Reiten quiver $\Gamma_{A}$ of an algebra $A$ admits a separating family $\mathcal{C}^{A}$ of almost cyclic coherent components if and only if $A$ is a generalized multicoil enlargement of a finite family $C_{1}, \ldots, C_{m}$ of concealed canonical algebras by an iterated application of admissible algebra operations of types (ad 1)-(ad 5) and their duals. Note that for such an algebra $A$, we have that $A$ is triangular, gl. $\operatorname{dim} A \leq 3$, and $\mathrm{pd}_{A} M \leq 2$ or id $A \leq 2$ for any module $M$ in ind $A$ (see [27, Corollary B and Theorem E]). Moreover, let $\Gamma_{A}=\mathcal{P}^{A} \cup \mathcal{C}^{A} \cup \mathcal{Q}^{A}$ be the induced decomposition of $\Gamma_{A}$. Then, by [27, Theorem C], there are uniquely determined quotient algebras $A^{(l)}=A_{1}^{(l)} \times \cdots \times A_{m}^{(l)}$ and $A^{(r)}=A_{1}^{(r)} \times \cdots \times A_{m}^{(r)}$ of $A$ which are the quasitilted algebras of canonical type such that $\mathcal{P}^{A}=\mathcal{P}^{A^{(l)}}$ and $\mathcal{Q}^{A}=\mathcal{Q}^{A^{(r)}}$. The algebras $A^{(l)}$ and $A^{(r)}$ are called the left and right quasitilted algebras of $A$. We note that $A^{(l)}$ and $A^{(r)}$ are tame if and only if $A^{(l)}$ and $A^{(r)}$ are products of tilted algebras of Euclidean type or tubular algebras. Moreover, by [27, Theorem $\mathrm{F}], A$ is tame if and only if $A^{(l)}$ and $A^{(r)}$ are tame.

In order to formulate our main results we need one more definition. Namely, if the sectional paths occurring in the definitions of the operations $(\operatorname{ad} 4),(\operatorname{fad} 4),\left(\operatorname{ad} 4^{*}\right),\left(\operatorname{fad} 4^{*}\right)$ come from a component or two components of the same connected algebra, then the modified translation quiver is said to be an exceptional configuration of modules (see Section 3 for details).

We mention that applying [30, Theorem 1.1] and [38, Theorem 4.1] we obtain that an algebra whose Auslander-Reiten quiver admits a separating family of almost cyclic coherent components with exceptional configurations of modules is not strongly simply connected (although its left and right quasitilted algebra can be strongly simply connected see Example 8.1 for details).

We are now in position to formulate the first main result of the paper.

Theorem 1.1 Let $A$ be an algebra with a separating family of almost cyclic coherent components in $\Gamma_{A}$ without exceptional configurations of modules. Then there are quotient algebras $A^{(l)}=A_{1}^{(l)} \times \cdots \times A_{m}^{(l)}$ and $A^{(r)}=A_{1}^{(r)} \times \cdots \times A_{m}^{(r)}$ of $A$ such that the following statements are equivalent:

(i) A is strongly simply connected.

(ii) For each $i \in\{1, \ldots, m\}, A_{i}^{(l)}$ and $A_{i}^{(r)}$ are strongly simply connected.

The following theorem is the second main result of the paper. 
Theorem 1.2 Let A be a tame algebra with a separating family of almost cyclic coherent components in $\Gamma_{A}$ without exceptional configurations of modules. Then there are quotient algebras $A^{(l)}=A_{1}^{(l)} \times \cdots \times A_{m}^{(l)}$ and $A^{(r)}=A_{1}^{(r)} \times \cdots \times A_{m}^{(r)}$ of $A$, where for each $i \in\{1, \ldots, m\}, A_{i}^{(l)}$ and $A_{i}^{(r)}$ are either the tilted algebras of Euclidean type or tubular algebras such that the following statements are equivalent:

(i) A is strongly simply connected.

(ii) For each $i \in\{1, \ldots, m\}, A_{i}^{(l)}$ and $A_{i}^{(r)}$ are strongly simply connected.

(iii) A does not contain a full convex subcategory which is hereditary of type $\widetilde{\mathbb{A}}_{n}$.

(iv) For each $i \in\{1, \ldots, m\}, A_{i}^{(l)}$ and $A_{i}^{(r)}$ do not contain a full convex subcategory which is hereditary of type $\widetilde{\mathbb{A}}_{n}$.

(v) For each $i \in\{1, \ldots, m\}$, the orbit graph of each directed component of $\Gamma_{A_{i}^{(l)}}$ and $\Gamma_{A_{i}^{(r)}}$ is a tree.

(vi) For each $i \in\{1, \ldots, m\}, A_{i}^{(l)}$ and $A_{i}^{(r)}$ satisfy the separation and coseparation conditions.

In order to present the first corollary, we need the class of cycle-finite algebras introduced by Assem and Skowroński in [9]. Let $A$ be an algebra. Recall that a cycle in a module category $\bmod A$ is a sequence

$$
M=M_{0} \stackrel{f_{1}}{\longrightarrow} M_{1} \rightarrow \cdots \rightarrow M_{r-1} \stackrel{f_{r}}{\longrightarrow} M_{r}=M
$$

of nonzero nonisomorphisms between indecomposable modules in $\bmod A$, and the cycle is said to be finite if the homomorphisms $f_{1}, \ldots, f_{r}$ do not belong to $\operatorname{rad}_{A}^{\infty}$. Then an algebra $A$ is said to be cycle-finite if all cycles in $\bmod A$ are finite. It is known (see [9]) that every cycle-finite algebra is tame.

As an application of Theorem 1.2 and results of [27, 40] we obtain the following corollary.

Corollary 1.3 Let A be a cycle-finite algebra with a separating family of almost cyclic coherent components in $\Gamma_{A}$ without exceptional configurations of modules. The following statements are equivalent:

(i) A is strongly simply connected.

(ii) A does not contain a full convex subcategory which is hereditary of type $\widetilde{\mathbb{A}}_{n}$.

Following Gerstenhaber [17], a one-parameter deformation of an algebra $A$ is a $k[[t]]-$ algebra structure on $k[[t]] \otimes_{k} A$ given by $f: A \otimes_{k} A \rightarrow k[[t]] \otimes_{k} A$ where $f(a \otimes b)=$ $a b+t \otimes f_{1}(a \otimes b)+t^{2} \otimes f_{2}(a \otimes b)+\cdots$ for $k$-bilinear morphisms $f_{i}: A \times A \rightarrow A$. Then the algebra $A$ is said to be analytically rigid if any one-parameter deformation of $A$ is isomorphic to the trivial one given by $f_{i}=0$ for $i \geq 1$. It was shown in [17] that oneparameter deformations of $A$ are related to low Hochschild cohomology spaces, $H^{i}(A)$, $1 \leq i \leq 3$. In fact, for a one-parameter deformation of $A$ given by $f: A \otimes_{k} A \rightarrow k[[t]] \otimes_{k} A$, the first $f_{i}$ different from zero defines an element of $H^{2}(A)$. Hence, if $H^{2}(A)=0$, then $A$ is analytically rigid. Moreover, if $H^{3}(A)=0$ and $A$ is analytically rigid, then $H^{2}(A)=0$.

For a connected concealed canonical algebra $C$ of tubular type $p_{C}=\left(p_{1}, \ldots, p_{t}\right)$ (see Section 2 for details), we set $t_{C}=t$. It is known that the class of concealed canonical algebras of type $\left(p_{1}, p_{2}\right)$ coincides with the class of hereditary algebras Euclidean 
types $\widetilde{\mathbb{A}}_{n}, n \geq 1$ (see [18]). For the definitions of generalized multicoil enlargement of a (not necessarily connected) concealed canonical algebra and admissible operations see Section 3.

As a direct consequence of Theorem 1.2, [30, Corollary 1.2] and the remark before Theorem 1.1 we obtain the following fact.

Corollary 1.4 Let A be a strongly simply connected tame algebra with a separating family of almost cyclic coherent components in $\Gamma_{A}$. The following statements are equivalent:

(i) A is analytically rigid.

(ii) $H^{2}(A)=0$.

(iii) $A$ is a tame generalized multicoil enlargement of a family $C_{1}, \ldots, C_{m}$ of connected concealed canonical algebras such that, for any $i \in\{1, \ldots, m\}, t_{C_{i}}=3$ and there is no admissible operation applied to modules from the mouth of stable tubes of rank one in $\Gamma_{C_{i}}$.

This paper is organized as follows. In Section 2 we recall some concepts and facts from representation theory, which are necessary for further considerations. Section 3 is devoted to describing some properties of almost cyclic coherent components of the Auslander-Reiten quivers of algebras, applied in the proofs of the preliminary results and the main theorems. In Section 4 we present and prove several results applied in the proof of the first main result of the paper. Sections 5, 6 and 7 are devoted to the proves of Theorems 1.1, 1.2 and Corollary 1.3, respectively. In Section 8 we present examples of tame algebras whose Auslander-Reiten quivers admit separating families of almost cyclic coherent components, illustrating Theorem 1.2. In Section 9 we present an application of Theorem 1.1. The aim of the final Section 10 is to present concluding remarks.

For basic background on the representation theory of algebras we refer to the books [7, 34-36] and for more details on algebras with separating families of Auslander-Reiten components and their representation theory to the survey article [29].

\section{Preliminaries}

\subsection{The separation condition}

Let $A$ be an algebra and $A \cong k Q_{A} / I$ be a presentation of $A$ as a bound quiver algebra. Then the algebra $A=k Q_{A} / I$ can equivalently be considered as a $k$-linear category, of which the object class $A_{0}$ is the set of points of $Q_{A}$, and the set of morphisms $A(x, y)$ from $x$ to $y$ is the quotient of the $k$-vector space $k Q_{A}(x, y)$ of all formal linear combinations of paths in $Q_{A}$ from $x$ to $y$ by the subspace $I(x, y)=k Q_{A}(x, y) \cap I$ (see [13]). A full subcategory $B$ of $A$ is called convex (in $A$ ) if any path in $A$ with source and target in $B$ lies entirely in $B$. For each vertex $v$ of $Q_{A}$ we denote by $S_{v}$ the corresponding simple $A$-module, and by $P_{v}$ (respectively, $I_{v}$ ) the projective cover (respectively, the injective envelope) of $S_{v}$.

Let $A$ be a triangular algebra. Recall that a vertex $v$ of $Q_{A}$ is called separating if the radical of $P_{v}$ is a direct sum of pairwise nonisomorphic indecomposable modules whose supports are contained in different connected components of the subquiver $Q(v)$ of $Q_{A}$ obtained by deleting all those vertices $u$ of $Q_{A}$ being the source of a path with target $v$ (including the trivial path from $v$ to $v$ ). Then, generalizing definition proposed in [12], we say that $A$ satisfy the separation condition if each vertex $v$ of $Q_{A}$ is separating. 


\subsection{One-point extensions and coextensions}

Frequently an algebra $A$ can be obtained from another algebra $B$ by a sequence of one-point extensions and one-point coextensions. Recall that the one-point extension of an algebra $B$ by a $B$-module $M$ is the matrix algebra

$$
B[M]=\left[\begin{array}{cc}
B & 0 \\
M & k
\end{array}\right]
$$

with the usual addition and multiplication of matrices. The quiver of $B[M]$ contains $Q_{B}$ as a convex subquiver and there is an additional (extension) point which is a source. $B[M]$ modules are usually identified with triples $(V, X, \varphi)$, where $V$ is a $k$-vector space, $X$ a $B$-module and $\varphi: V \rightarrow \operatorname{Hom}_{B}(M, X)$ a $k$-linear map. A $B[M]$-linear map $(V, X, \varphi) \rightarrow$ $\left(V^{\prime}, X^{\prime}, \varphi^{\prime}\right)$ is then identified with a pair $(f, g)$, where $f: V \rightarrow V^{\prime}$ is $k$-linear, $g: X \rightarrow X^{\prime}$ is $B$-linear and $\varphi^{\prime} f=\operatorname{Hom}_{B}(M, g) \varphi$. One defines dually the one-point coextension $[M] B$ of $B$ by $M$ (see [34]).

\subsection{Tameness}

Let $A$ be an algebra and $K[x]$ the polynomial algebra in one variable $x$. Following [16] $A$ is said to be tame if, for any dimension $d$, there exists a finite number of $K[x]-A$-bimodules $M_{i}, 1 \leq i \leq n_{d}$, which are finitely generated and free as left $K[x]$-modules, and all but a finite number of isoclasses of indecomposable $A$-modules of dimension $d$ are of the form $K[x] /(x-\lambda) \otimes_{K[x]} M_{i}$ for some $\lambda \in K$ and some $i \in\left\{1, \ldots, n_{d}\right\}$. Let $\mu_{A}(d)$ be the least number of $K[x]-A$-bimodules $M_{i}$ satisfying the above condition for $d$. Then $A$ is said to be domestic [34] (respectively, polynomial growth [37]) if there exists a positive integer $m$ such that $\mu_{A}(d) \leq m$ (respectively, $\mu_{A}(d) \leq d^{m}$ ) for any $d \geq 1$.

\subsection{Simple connectedness}

Let $(Q, I)$ be a connected bound quiver. A relation $\varrho=\sum_{i=1}^{m} \lambda_{i} w_{i} \in I(x, y)$ is minimal if $m \geq 2$ and, for any nonempty proper subset $J \subset\{1, \ldots, m\}$, we have $\sum_{j \in J} \lambda_{j} w_{j} \notin$ $I(x, y)$, where for each $i \in\{1, \ldots, m\} \lambda_{i}$ is a non-zero scalar and $w_{i}$ is a path of length at least two from $x$ to $y$. We denote by $\alpha^{-1}$ the formal inverse of an arrow $\alpha \in Q_{1}$. A walk in $Q$ from $x$ to $y$ is a formal composition $\alpha_{1}^{\varepsilon_{1}} \alpha_{2}^{\varepsilon_{2}} \ldots \alpha_{t}^{\varepsilon_{t}}$ (where $\alpha_{i} \in Q_{1}$ and $\varepsilon_{i} \in\{-1,1\}$ for all $i$ ) with source $x$ and target $y$. We denote by $e_{x}$ the trivial path at $x$. Let $\sim$ be the least equivalence relation on the set of all walks in $Q$ such that:

(a) If $\alpha: x \rightarrow y$ is an arrow, then $\alpha^{-1} \alpha \sim e_{y}$ and $\alpha \alpha^{-1} \sim e_{x}$.

(b) If $\varrho=\sum_{i=1}^{m} \lambda_{i} w_{i}$ is a minimal relation, then $w_{i} \sim w_{j}$ for all $i, j$.

(c) If $u \sim v$, then $w u w^{\prime} \sim w v w^{\prime}$ whenever these compositions make sense.

Let $x \in Q_{0}$ be arbitrary. The set $\pi_{1}(Q, I, x)$ of equivalence classes $\tilde{u}$ of closed walks $u$ starting and ending at $u$ has a group structure defined by the operation $\tilde{u} \cdot \widetilde{v}=\widetilde{u v}$. Since $Q$ is connected, $\pi_{1}(Q, I, x)$ does not depend on the choice of $x$. We denote it by $\pi_{1}(Q, I)$ and call it the fundamental group of $(Q, I)$.

Let $A \cong k Q_{A} / I$ be a presentation of a triangular algebra $A$ as a bound quiver algebra. The fundamental group $\pi_{1}\left(Q_{A}, I\right)$ depends essentially on $I$, so is not an invariant of $A$. A triangular algebra $A$ is called simply connected if, for any presentation $A \cong k Q_{A} / I$ of $A$ as a bound quiver algebra, the fundamental group $\pi_{1}\left(Q_{A}, I\right)$ of $\left(Q_{A}, I\right)$ is trivial [8]. 


\subsection{Strong simple connectedness}

Let $A$ be a triangular algebra. Following [38] $A$ is called a strongly simply connected if it satisfies one of the following equivalent conditions:

(a) Any full convex subcategory of $A$ is simply connected.

(b) Any full convex subcategory of $A$ satisfies the separation condition.

(c) Any full convex subcategory of $A$ satisfies the coseparation condition.

(d) The first Hochschild cohomology space $H^{1}(B)$ of any full convex subcategory $B$ of $A$ vanishes.

In the proof of Theorem 1.1 we will use a characterization of strongly simply connected algebras established by Assem and Liu [4]. We now recall the necessary definitions and the appropriate theorem. Let $Q$ be a locally finite quiver without oriented cycles. A contour $(p, q)$ in $Q$ from $x$ to $y$ consists of a pair of non-trivial paths from $x$ to $y$. It is interlaced if $p, q$ have a common point besides $x$ and $y$. A contour $(p, q)$ is irreducible if there exists no sequence of paths $p=p_{0}, p_{1}, \ldots, p_{m}=q$ in $Q$ from $x$ to $y$ such that, for each $i \in\{1, \ldots, m\}$, the contour $\left(p_{i-1}, p_{i}\right)$ is interlaced. Let $C$ be a simple cycle which is not a contour, and let $\sigma(C)$ denote the number of sources in $C$. Then $C$ is reducible if there exist $x, y$ on $C$ and a path $p: x \rightarrow \cdots \rightarrow y$ in $Q$ such that if $w_{1}$ and $w_{2}$ denote the subwalks of $C$ from $x$ to $y$ (so that $C=w_{1} w_{2}^{-1}$ ), then $w_{1} p^{-1}$ and $w_{2} p^{-1}$ are cycles and $\sigma\left(w_{1} p^{-1}\right)<\sigma(C), \sigma\left(w_{2} p^{-1}\right)<\sigma(C)$. A cycle $C$ is irreducible if either it is an irreducible contour, or it is not a contour, but it is not reducible in the above sense. Let $I$ be an admissible ideal of $k Q$. Finally, a contour $(p, q)$ from $x$ to $y$ is naturally contractible in a bound quiver $(Q, I)$ if there exists a sequence of paths $p=p_{0}, p_{1}, \ldots, p_{m}=q$ in $Q$ such that, for each $i \in\{1, \ldots, m\}$, the paths $p_{i-1}$ and $p_{i}$ have subpaths $q_{i-1}$ and $q_{i}$, respectively, which are involved in the same minimal relation in $(Q, I)$.

We need the following result proved in [4, Theorem 1.3].

Theorem 2.6 A triangular algebra $A$ is strongly simply connected if and only if, for any presentation $A \cong k Q_{A} / I$ of $A$ as a bound quiver algebra, each irreducible cycle in $Q_{A}$ is an irreducible contour, and each irreducible contour in $Q_{A}$ is naturally contractible in $\left(Q_{A}, I\right)$.

\section{Almost cyclic coherent Auslander-Reiten components}

It has been proved in [26, Theorem A] that a connected component $\Gamma$ of an AuslanderReiten quiver $\Gamma_{A}$ of an algebra $A$ is almost cyclic and coherent if and only if $\Gamma$ is a generalized multicoil, that is, can be obtained, as a translation quiver, from a finite family of stable tubes by a sequence of operations called admissible. We recall briefly the generalized multicoil enlargements of algebras from [27, Section 3].

Given a generalized standard component $\Gamma$ of $\Gamma_{A}$, and an indecomposable module $X$ in $\Gamma$, the support $\mathcal{S}(X)$ of the functor $\left.\operatorname{Hom}_{A}(X,-)\right|_{\Gamma}$ is the $k$-linear category defined as follows [10]. Let $\mathcal{H}_{X}$ denote the full subcategory of $\Gamma$ consisting of the indecomposable modules $M$ in $\Gamma$ such that $\operatorname{Hom}_{A}(X, M) \neq 0$, and $\mathcal{I}_{X}$ denote the ideal of $\mathcal{H}_{X}$ consisting of the morphisms $f: M \rightarrow N$ (with $M, N$ in $\left.\mathcal{H}_{X}\right)$ such that $\operatorname{Hom}_{A}(X, f)=0$. We define $\mathcal{S}(X)$ to be the quotient category $\mathcal{H}_{X} / \mathcal{I}_{X}$. Following the above convention, we usually identify the $k$-linear category $\mathcal{S}(X)$ with its quiver.

Recall that a module $X$ in $\bmod A$ is called a brick if $\operatorname{End}_{A}(X) \cong k$. 
Let $A$ be an algebra and $\Gamma$ be a family of generalized standard infinite components of $\Gamma_{A}$. For an indecomposable brick $X$ in $\Gamma$, called the pivot, five admissible operations are defined, depending on the shape of the support $\mathcal{S}(X)$ of the functor $\left.\operatorname{Hom}_{A}(X,-)\right|_{\Gamma}$. These admissible operations yield in each case a modified algebra $A^{\prime}$ such that the modified translation quiver $\Gamma^{\prime}$ is a family of generalized standard infinite components in the Auslander-Reiten quiver $\Gamma_{A^{\prime}}$ of $A^{\prime}$ (see [26, Section 2] or [29, Section 4] for the figures illustrating the modified translation quiver $\Gamma^{\prime}$ ).

(ad 1) Assume $\mathcal{S}(X)$ consists of an infinite sectional path starting at $X$ :

$$
X=X_{0} \rightarrow X_{1} \rightarrow X_{2} \rightarrow \cdots
$$

Let $t \geq 1$ be a positive integer, $D$ be the full $t \times t$ lower triangular matrix algebra, and $Y_{1}, \ldots$, $Y_{t}$ denote the indecomposable injective $D$-modules with $Y=Y_{1}$ the unique indecomposable projective-injective $D$-module. We set $A^{\prime}=(A \times D)[X \oplus Y]$. In this case, $\Gamma^{\prime}$ is obtained by inserting in $\Gamma$ the rectangle consisting of the modules $Z_{i j}=\left(k, X_{i} \oplus Y_{j},\left[\begin{array}{l}1 \\ 1\end{array}\right]\right)$ for $i \geq 0$, $1 \leq j \leq t$, and $X_{i}^{\prime}=\left(k, X_{i}, 1\right)$ for $i \geq 0$. If $t=0$ we set $A^{\prime}=A[X]$ and the rectangle reduces to the sectional path consisting of the modules $X_{i}^{\prime}, i \geq 0$.

(ad 2) Suppose that $\mathcal{S}(X)$ admits two sectional paths starting at $X$, one infinite and the other finite with at least one arrow:

$$
Y_{t} \leftarrow \cdots \leftarrow Y_{2} \leftarrow Y_{1} \leftarrow X=X_{0} \rightarrow X_{1} \rightarrow X_{2} \rightarrow \cdots
$$

where $t \geq 1$. In particular, $X$ is necessarily injective. We set $A^{\prime}=A[X]$. In this case, $\Gamma^{\prime}$ is obtained by inserting in $\Gamma$ the rectangle consisting of the modules $Z_{i j}=\left(k, X_{i} \oplus Y_{j},\left[\begin{array}{l}1 \\ 1\end{array}\right]\right)$ for $i \geq 1,1 \leq j \leq t$, and $X_{i}^{\prime}=\left(k, X_{i}, 1\right)$ for $i \geq 0$.

(ad 3) Assume $\mathcal{S}(X)$ is the mesh-category of two parallel sectional paths:

$$
\begin{array}{ccc}
Y_{1} & \rightarrow Y_{2} \rightarrow \cdots \rightarrow Y_{t} \\
\uparrow & \uparrow & \uparrow \\
X=X_{0} & \rightarrow X_{1} \rightarrow \cdots \rightarrow X_{t-1} \rightarrow X_{t} \rightarrow \cdots
\end{array}
$$

with the upper sectional path finite and $t \geq 2$. In particular, $X_{t-1}$ is necessarily injective. Moreover, we consider the translation quiver $\bar{\Gamma}$ of $\Gamma$ obtained by deleting the arrows $Y_{i} \rightarrow$ $\tau_{A}^{-1} Y_{i-1}$. We assume that the union $\widehat{\Gamma}$ of connected components of $\bar{\Gamma}$ containing the modules $\tau_{A}^{-1} Y_{i-1}, 2 \leq i \leq t$, is a finite translation quiver. Then $\bar{\Gamma}$ is a disjoint union of $\widehat{\Gamma}$ and a cofinite full translation subquiver $\Gamma^{*}$, containing the pivot $X$. We set $A^{\prime}=A[X]$. In this case, $\Gamma^{\prime}$ is obtained from $\Gamma^{*}$ by inserting the rectangle consisting of the modules $Z_{i j}=$ $\left(k, X_{i} \oplus Y_{j},\left[\begin{array}{l}1 \\ 1\end{array}\right]\right)$ for $i \geq 1,1 \leq j \leq i$, and $X_{i}^{\prime}=\left(k, X_{i}, 1\right)$ for $i \geq 0$.

(ad 4) Suppose that $\mathcal{S}(X)$ consists an infinite sectional path, starting at $X$

$$
X=X_{0} \rightarrow X_{1} \rightarrow X_{2} \rightarrow \cdots \text { and } Y=Y_{1} \rightarrow Y_{2} \rightarrow \cdots \rightarrow Y_{t}
$$

with $t \geq 1$, be a finite sectional path in $\Gamma_{A}$. Let $r$ be a positive integer. Moreover, we consider the translation quiver $\bar{\Gamma}$ of $\Gamma$ obtained by deleting the arrows $Y_{i} \rightarrow \tau_{A}^{-1} Y_{i-1}$. We assume that the union $\widehat{\Gamma}$ of connected components of $\bar{\Gamma}$ containing the vertices $\tau_{A}^{-1} Y_{i-1}$, $2 \leq i \leq t$, is a finite translation quiver. Then $\bar{\Gamma}$ is a disjoint union of $\widehat{\Gamma}$ and a cofinite full translation subquiver $\Gamma^{*}$, containing the pivot $X$. For $r=0$ we set $A^{\prime}=A[X \oplus Y]$. In this case, $\Gamma^{\prime}$ is obtained from $\Gamma^{*}$ by inserting the rectangle consisting of the modules $Z_{i j}=\left(k, X_{i} \oplus Y_{j},\left[\begin{array}{l}1 \\ 1\end{array}\right]\right)$ for $i \geq 0,1 \leq j \leq t$, and $X_{i}^{\prime}=\left(k, X_{i}, 1\right)$ for $i \geq 0$. 
For $r \geq 1$, let $G$ be the full $r \times r$ lower triangular matrix algebra, $U_{1, t+1}, U_{2, t+1}$, $\ldots, U_{r, t+1}$ denote the indecomposable projective $G$-modules, $U_{r, t+1}, U_{r, t+2}, \ldots, U_{r, t+r}$ denote the indecomposable injective $G$-modules, with $U_{r, t+1}$ the unique indecomposable projective-injective $G$-module. We set define the

$$
A^{\prime}=\left[\begin{array}{cccccc}
A & 0 & 0 & \ldots & 0 & 0 \\
Y & k & 0 & \ldots & 0 & 0 \\
Y & k & k & \ldots & 0 & 0 \\
\vdots & \vdots & \vdots & \ddots & \vdots & \vdots \\
Y & k & k & \ldots & k & 0 \\
X \oplus Y & k & k & \ldots & k & k
\end{array}\right]
$$

with $r+2$ columns and rows. In this case, $\Gamma^{\prime}$ is obtained from $\Gamma^{*}$ by inserting the following modules

$$
\begin{aligned}
& U_{s l}= \begin{cases}\left(k, Y_{l}, 1\right) & \text { for } s=1,1 \leq l \leq t, \\
\left(k, U_{s, l-1}, 1\right) & \text { for } 2 \leq s \leq r, 1 \leq l<t+s, \\
(k, 0,0) & \text { for } 2 \leq s \leq r, l=t+s\end{cases} \\
& Z_{i j}=\left(k, X_{i} \oplus U_{r j},\left[\begin{array}{l}
1 \\
1
\end{array}\right]\right) \text { for } i \geq 0,1 \leq j \leq t+r,
\end{aligned}
$$

and $X_{i}^{\prime}=\left(k, X_{i}, 1\right)$ for $i \geq 0$. In the above formulas $U_{s l}$ is treated as a module over the algebra $A_{s}=A_{s-1}\left[U_{s-1,1}\right]$, where $A_{0}=A$ and $U_{01}=Y$ (in other words $A_{s}$ is an algebra consisting of matrices obtained from the matrices belonging to $A^{\prime}$ by choosing the first $s+1$ rows and columns).

We note that the quiver $Q_{A^{\prime}}$ of $A^{\prime}$ is obtained from the quiver of the double one-point extension $A[X][Y]$ by adding a path of length $r+1$ with source at the extension vertex of $A[X]$ and sink at the extension vertex of $A[Y]$.

To the definition of the next admissible operation we need also the finite versions of the admissible operations ( $\operatorname{ad} 1),(\operatorname{ad} 2),(\operatorname{ad} 3),(\operatorname{ad} 4)$, which we denote by (fad 1), (fad 2), (fad 3$)$ and (fad 4), respectively. In order to obtain these operations we replace all infinite sectional paths of the form $X_{0} \rightarrow X_{1} \rightarrow X_{2} \rightarrow \cdots$ (in the definitions of (ad 1), (ad 2), (ad 3), (ad 4)) by the finite sectional paths of the form $X_{0} \rightarrow X_{1} \rightarrow X_{2} \rightarrow \cdots \rightarrow X_{s}$. For the operation (fad 1) $s \geq 0$, for (fad 2) and (fad 4) $s \geq 1$, and for (fad 3) $s \geq t-1$. In all above operations $X_{s}$ is injective (see the figures for (fad 1)-(fad 4) in [26, Section 2] or [29, Section 4]).

(ad 5) We define the modified algebra $A^{\prime}$ of $A$ to be the iteration of the extensions described in the definitions of the admissible operations $(\operatorname{ad} 1),(\operatorname{ad} 2),(\operatorname{ad} 3),(\operatorname{ad} 4)$, and their finite versions corresponding to the operations (fad 1), (fad 2), (fad 3) and (fad 4). In this case, $\Gamma^{\prime}$ is obtained in the following three steps: first we are doing on $\Gamma$ one of the operations (fad 1), (fad 2) or (fad 3), next a finite number (possibly zero) of the operation (fad 4) and finally the operation ( $\mathrm{ad} 4$ ), and in such a way that the sectional paths starting from all the new projective modules have a common cofinite (infinite) sectional subpath. By an (ad 5)pivot we mean an indecomposable brick $X$ from the last (ad 4) operation used in the whole process of creating (ad 5).

Moreover, together with each of the admissible operations ( $\mathrm{ad} \mathrm{1)-(ad} \mathrm{5),} \mathrm{we} \mathrm{consider}$ its dual, denoted by $\left(\mathrm{ad} 1^{*}\right)-\left(\operatorname{ad~} 5^{*}\right)$. These dual operations are also called admissible. Following [26] a connected translation quiver $\Gamma$ is said to be a generalized multicoil if $\Gamma$ can 
be obtained from a finite family $\mathcal{T}_{1}, \mathcal{T}_{2}, \ldots, \mathcal{T}_{s}$ of stable tubes by an iterated application of admissible operations $(\operatorname{ad} 1),\left(\operatorname{ad} 1^{*}\right),(\operatorname{ad} 2),\left(\operatorname{ad} 2^{*}\right),(\operatorname{ad} 3),\left(\operatorname{ad} 3^{*}\right),(\operatorname{ad} 4),\left(\operatorname{ad} 4^{*}\right),(\operatorname{ad} 5)$ or $\left(\operatorname{ad} 5^{*}\right)$. If $s=1$, such a translation quiver $\Gamma$ is said to be a generalized coil. The admissible operations of types $(\operatorname{ad} 1)-(\operatorname{ad} 3),\left(\operatorname{ad~} 1^{*}\right)-\left(\operatorname{ad~} 3^{*}\right)$ have been introduced in [9-11], and the admissible operations ( $\mathrm{ad} 4)$ and $\left(\mathrm{ad} 4^{*}\right)$ for $r=0$ in [24].

Finally, let $C$ be a (not necessarily connected) concealed canonical algebra (see [21]) and $\mathcal{T}^{C}$ a separating family of stable tubes of $\Gamma_{C}$. Following [27] we say that an algebra $A$ is a generalized multicoil enlargement of $C$ using modules from $\mathcal{T}^{C}$ if there exists a sequence of algebras $C=A_{0}, A_{1}, \ldots, A_{n}=A$ such that $A_{i+1}$ is obtained from $A_{i}$ by an admissible operation of one of the types $(\operatorname{ad} 1)-(\operatorname{ad} 5),\left(\operatorname{ad~} 1^{*}\right)-\left(\operatorname{ad~} 5^{*}\right)$ performed either on stable tubes of $\mathcal{T}^{A_{i}}$, or on generalized multicoils obtained from stable tubes of $\mathcal{T}^{A_{i}}$ by means of operations done so far. The sequence $C=A_{0}, A_{1}, \ldots, A_{n}=A$ is then called an admissible sequence for $A$. Observe that this definition extends the concept of a coil enlargement of a concealed canonical algebra introduced in [11]. We note that a generalized multicoil enlargement $A$ of $C$ invoking only admissible operations of type (ad 1) (respectively, of type $\left.\left(\operatorname{ad} 1^{*}\right)\right)$ is a tubular extension (respectively, tubular coextension) of $C$ in the sense of [34]. An algebra $A$ is said to be a generalized multicoil algebra if $A$ is a connected generalized multicoil enlargement of a product $C$ of connected concealed canonical algebras.

Let $A$ be an algebra. It has been proved in [27, Theorem A] that $\Gamma_{A}$ admits a separating family of almost cyclic coherent components if and only if $A$ is a generalized multicoil enlargement of a concealed canonical algebra $C$.

Let us note that the class of algebras with generalized standard almost cyclic coherent Auslander-Reiten components is large (see [28, Proposition 2.9] and the following comments).

We also note that the class of tubular extension (respectively, tubular coextension) of concealed canonical algebras coincides with the class of algebras having a separating family of ray tubes (respectively, coray tubes) in their Auslander-Reiten quiver (see [21, 23]). Moreover, these algebras are quasitilted algebras of canonical type. See also [27, Theorem C] describing the structure of the module category of an algebra with a separating family of almost cyclic coherent Auslander-Reiten components.

\section{Preliminary results}

\subsection{Branch extensions and coextensions}

Let $A$ be an algebra and $A \cong k Q_{A} / I$ be a presentation of $A$ as a bound quiver algebra. For a given vertex $v$ in $Q_{A}$, we denote by $v \rightarrow$ (respectively, by $\rightarrow v$ ) the set of all arrows of the quiver $Q_{A}$ starting at $v$ (respectively, terminating at $v$ ). Let now $K$ be a branch at a vertex $v \in Q_{A}$ and $E \in \bmod A$. Recall that the branch extension $A[E, K]$ by the branch $K$ [34, (4.4)] is constructed in the following way: to the one-point extension $A[E]$ with extension vertex $w$ (that is, $\operatorname{rad} P_{w}=E$ ) we add the branch $K$ by identifying the vertices $v$ and $w$. If $E_{1}, \ldots, E_{n} \in \bmod A$ and $K_{1}, \ldots, K_{n}$ is a set of branches, then the branch extension $A\left[E_{i}, K_{i}\right]_{i=1}^{n}$ is defined inductively as: $A\left[E_{i}, K_{i}\right]_{i=1}^{n}=\left(A\left[E_{i}, K_{i}\right]_{i=1}^{n-1}\right)\left[E_{n}, K_{n}\right]$. The concept of branch coextension is defined dually.

Lemma 4.2 Let $A$ be a generalized multicoil enlargement of $C=C_{1} \times \cdots \times C_{m}$, where $C_{1}, \ldots, C_{m}$ is a family of tame concealed algebras. Moreover, let $C=A_{0}, \ldots, A_{p}=$ 
$A^{(l)}, A_{p+1}, \ldots, A_{n}=A$ be an admissible sequence for $A, j \geq p, X \in$ ind $A_{j}$ be an (ad 1)pivot, $A_{j+1}$ be the modified algebra of $A_{j}$, and $v$ be the corresponding extension point. Then the following statements hold.

(i) If there is a vertex $u \in A^{(l)} \backslash A^{(r)}$ such that each $\alpha \in v \rightarrow$ is the starting point of a non-zero path $\omega_{\alpha} \in A(v, u)$, then:

(a) The vertex $u$ is unique.

(b) There are at least two different arrows in $v \rightarrow$.

(c) If $\alpha, \beta \in v^{\rightarrow}$, and $\alpha \neq \beta$, then $\omega_{\alpha}-\omega_{\beta} \in I$.

(ii) If $\left.X\right|_{C_{i}}=0$ for any $i \in\{1, \ldots, m\}$, then $X$ is uniserial.

Proof Since $X$ is an (ad 1)-pivot, the support $\mathcal{S}(X)$ consists of an infinite sectional path $X=X_{0} \rightarrow X_{1} \rightarrow X_{2} \rightarrow \cdots$ starting at $X$. Let $t \geq 1$ be a positive integer, $D$ be the full $t \times t$ lower triangular matrix algebra, and $Y_{1}, \ldots, Y_{t}$ be the indecomposable injective $D$ modules with $Y_{1}$ the unique indecomposable projective-injective $D$-module (see definition of $(\operatorname{ad} 1))$.

(i) We know from [27, Section 4] that $A^{(l)}$ is a unique maximal convex branch coextension of $C=C_{1} \times \cdots \times C_{m}$ inside $A$, that is, $A^{(l)}=B_{1}^{(l)} \times \cdots \times B_{m}^{(l)}$, where $B_{i}^{(l)}$ is a unique maximal convex branch coextension of $C_{i}$ inside $A, i \in\{1, \ldots, m\}$. More precisely, $B_{i}^{(l)}={ }_{j=1}^{t_{i}}\left[K_{j}, E_{j}\right] C_{i}$, where $K_{1}, \ldots, K_{t_{i}}$ are branches, $i \in\{1, \ldots, m\}$. Assume that there is a vertex $u \in A^{(l)} \backslash A^{(r)}$ such that each $\alpha \in v \rightarrow$ is the starting point of a nonzero path $\omega_{\alpha} \in A(v, u)$. Then there exists $s \in\{1, \ldots, m\}$ such that $u \in B_{s}^{(l)}$. Moreover, $A_{j+1}=\left(A_{j} \times D\right)\left[X \oplus Y_{1}\right]$ and the bound quiver $Q_{A_{j+1}} \mid \operatorname{Supp} X$ is of the form

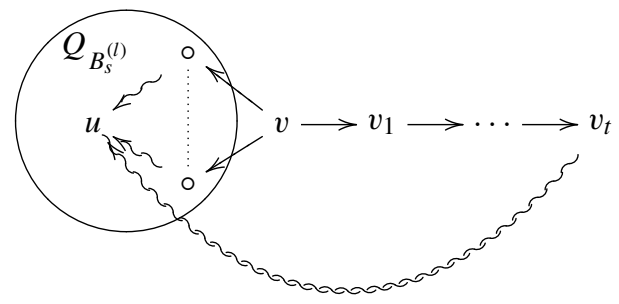

where $v_{1}, \ldots, v_{t}$ are the points in the quiver $Q_{A_{j+1}}$ of $A_{j+1}$ corresponding to the new indecomposable projective $A_{j+1}$-modules. Then $A_{j+1}$ is the extension of $B_{s}^{(l)}$ at $X$ by the extension branch $K$ consisting of the points $v, v_{1}, \ldots, v_{t}$, that is, we have $A_{j+1}=$ $A_{j}[X, K]$. Since $u$ does not belong to $A^{(r)}$ and for any $\alpha \in v \rightarrow$ it is the starting point of a non-zero path $\omega_{\alpha} \in A(v, u)$, we get that $u$ is the coextension point of the admissible operation $\left(\operatorname{ad} 2^{*}\right)$ or $\left(\operatorname{ad~} 3^{*}\right)$. By [11, Lemma 3.1] the admissible operations $\left(\operatorname{ad~} 2^{*}\right)$ and $\left(\right.$ ad $\left.3^{*}\right)$ commute with (ad 1), so we can apply (ad $\left.2^{*}\right)$ after (ad 1) (respectively, $\left(\operatorname{ad~} 3^{*}\right)$ after (ad 1)). Using now [11, Lemma 3.3] (respectively, [11, Lemma 3.4]), we are able to replace $(\operatorname{ad} 1)$ followed by $\left(\operatorname{ad~} 2^{*}\right)$ (respectively, (ad 1) followed by $\left(\operatorname{ad~} 3^{*}\right)$ ) by an operation of type $\left(\operatorname{ad~} 1^{*}\right)$ followed by an operation of type (ad 2) (respectively, $\left(\operatorname{ad~} 1^{*}\right)$ followed by an operation of type ( $\mathrm{ad} 3)$ ). Therefore, the statements (a), (b) and (c) follow from Lemma 4.3.

(ii) A case by case inspection shows that $X$ is either simple module or the support of $X$ is a linearly ordered quiver of type $\mathbb{A}_{t}$. 
Lemma 4.3 Let $A$ be a generalized multicoil enlargement of $C=C_{1} \times \cdots \times C_{m}$, where $C_{1}, \ldots, C_{m}$ is a family of tame concealed algebras. Moreover, let $C=A_{0}, \ldots, A_{p}=$ $A^{(l)}, A_{p+1}, \ldots, A_{n}=A$ be an admissible sequence for $A, j \geq p, X \in \operatorname{ind} A_{j}$ be an $(\operatorname{ad} 2)$ or (ad 3)-pivot, and $A_{j+1}$ be the modified algebra of $A_{j}$. If $v$ is the corresponding extension point then there is a unique vertex $u \in A^{(l)} \backslash A^{(r)}$ that satisfies:

(i) Each $\alpha \in v \rightarrow$ is the starting point of a non-zero path $\omega_{\alpha} \in A(v, u)$.

(ii) There are at least two different arrows in $v \rightarrow$. Moreover, if $\alpha, \beta \in v \rightarrow$, and $\alpha \neq \beta$, then $\omega_{\alpha}-\omega_{\beta} \in I$.

Proof Again, we know from [27, Section 4] that $A^{(l)}$ is a unique maximal convex branch coextension of $C=C_{1} \times \cdots \times C_{m}$ inside $A$, that is, $A^{(l)}=B_{1}^{(l)} \times \cdots \times B_{m}^{(l)}$, where $B_{i}^{(l)}$ is a unique maximal convex branch coextension of $C_{i}$ inside $A, i \in\{1, \ldots, m\}$. More precisely, $B_{i}^{(l)}={ }_{j=1}^{t_{i}}\left[K_{j}, E_{j}\right] C_{i}$, where $K_{1}, \ldots, K_{t_{i}}$ are branches, $i \in\{1, \ldots, m\}$. Then there exists $s \in\{1, \ldots, m\}$ such that $u \in B_{s}^{(l)}$ and $A_{j+1}=A_{j}[X]$. If $X$ is an (ad 2)-pivot (respectively, (ad 3)-pivot), then in the sequence of earlier admissible operations, there is an operation of type $\left(\operatorname{ad~} 1^{*}\right)$ or $\left(\operatorname{ad~} 5^{*}\right)$ which contains an operation $\left(\mathrm{fad} 1^{*}\right)$ which gives rise to the pivot $X$ of $(\operatorname{ad} 2)$ (respectively, to the pivot $X$ of $(\operatorname{ad} 3)$ and to the modules $Y_{1}, \ldots, Y_{t}$ in the support of $\operatorname{Hom}_{A}(X,-)$ restricted to the generalized multicoil containing $X$ - see definition of $(\operatorname{ad} 3))$. The operations done after must not affect the support of $\operatorname{Hom}_{A}(X,-)$ restricted to the generalized multicoil containing $X$. Note that in general, in the sequence of earlier admissible operations can be an operation of type ( $\mathrm{ad} 5)$ which contains an operation (fad 4) which gives rise to the pivot $X$ of (ad 2) (respectively, to the pivot $X$ of $(\operatorname{ad} 3)$ ) but from Lemma [27, Lemma 3.10] this case can be reduced to $\left(\mathrm{ad} 5^{*}\right)$ which contains an operation $\left(\operatorname{fad} 1^{*}\right)$.

Let $X$ be an (ad 2)-pivot, $A_{j+1}=A_{j}[X]$, and $u, u_{1}, \ldots, u_{t}\left(X=I_{u}\right)$ be the points in the quiver $Q_{A_{j}}$ of $A_{j}$ corresponding to the new indecomposable injective $A_{j}$-modules obtained after performing the above admissible operation $\left(\mathrm{ad} 1^{*}\right)$ or the operation $\left(\mathrm{fad} 1^{*}\right)$. The bound quiver $Q_{A_{j+1}}$ of $A_{j+1}$ is of the form

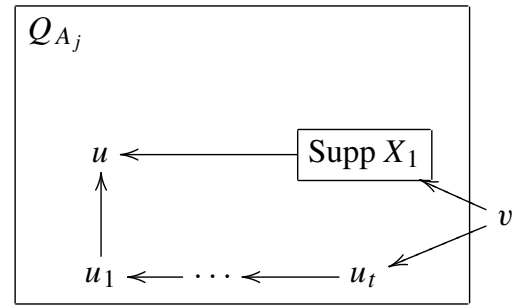

where $X_{1}$ is the immediate successor of $X$ on the infinite sectional path in $\mathcal{S}(X)$ (see definition of $(\operatorname{ad} 2))$.

Let now $X$ be an (ad 3)-pivot, $A_{j+1}=A_{j}[X]$, and assume that we had $r$ consecutive admissible operations of types $\left(\mathrm{ad} 1^{*}\right)$ or (fad $\left.1^{*}\right)$, the first of which had $X_{t}$ as a pivot, and these admissible operations built up a branch $K$ in $A_{j}$ with points $u, u_{1}, \ldots, u_{t}$ in $Q_{A_{j}}$, so that $X_{t-1}$ and $Y_{t}$ are the indecomposable injective $A_{j}$-modules corresponding 
respectively to $u$ and $u_{1}$, and both $Y_{1}$ and $\tau_{A_{j}}^{-1} Y_{1}$ are coray modules in the generalized multicoil containing the (ad 3)-pivot $X$. The bound quiver $Q_{A_{j+1}}$ of $A_{j+1}$ is of the form

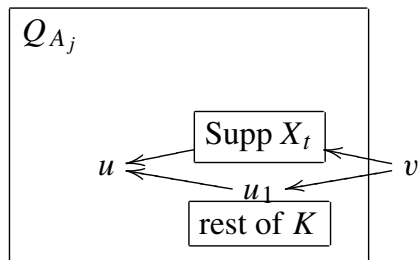

where $X, X_{t-1}, X_{t}, Y_{1}$ and $Y_{t}$ are as in the definition of ( $\left.\operatorname{ad} 3\right)$. Now, the statements follows from the proof of [14, Lemma 2.4].

Lemma 4.4 Let $A$ be a generalized multicoil enlargement of $C=C_{1} \times \cdots \times C_{m}$, where $C_{1}, \ldots, C_{m}$ is a family of tame concealed algebras. Moreover, let $C=A_{0}, \ldots, A_{p}=$ $A^{(l)}, A_{p+1}, \ldots, A_{n}=A$ be an admissible sequence for $A, j \geq p, X \in$ ind $A_{j}$ be an (ad 4) or (ad 5)-pivot, $A_{j+1}$ be the modified algebra of $A_{j}$, and $v$ be the corresponding extension point. If there is a vertex $u \in A^{(l)} \backslash A^{(r)}$ such that for pairwise different arrows $\alpha_{1}, \ldots, \alpha_{q} \in v \rightarrow, q \geq 2$ there are paths $\omega_{\alpha_{1}}, \ldots, \omega_{\alpha_{q}} \in A(v, u)$, then for arbitrary $f, g \in$ $\{1, \ldots, q\}, f \neq g$, one of the following cases holds:

(i) At least one of $\omega_{\alpha_{f}}, \omega_{\alpha_{g}}$ is zero path.

(ii) The paths $\omega_{\alpha_{f}}, \omega_{\alpha_{g}}$ are non-zero and $\omega_{\alpha_{f}}-\omega_{\alpha_{g}} \in I$.

Proof It follows from [27, Section 4] that $A^{(l)}$ is a unique maximal convex branch coextension of $C=C_{1} \times \cdots \times C_{m}$ inside $A$, that is, $A^{(l)}=B_{1}^{(l)} \times \cdots \times B_{m}^{(l)}$, where $B_{i}^{(l)}$ is a unique maximal convex branch coextension of $C_{i}$ inside $A, i \in\{1, \ldots, m\}$. More precisely, $B_{i}^{(l)}={ }_{j=1}^{t_{i}}\left[K_{j}, E_{j}\right] C_{i}$, where $K_{1}, \ldots, K_{t_{i}}$ are branches, $i \in\{1, \ldots, m\}$. Assume that there is a vertex $u \in A^{(l)} \backslash A^{(r)}$ such that for pairwise different arrows $\alpha_{1}, \ldots, \alpha_{q} \in v^{\rightarrow}, q \geq 2$, there are paths $\omega_{\alpha_{1}}, \ldots, \omega_{\alpha_{q}} \in A(v, u)$. Then there exists $s \in\{1, \ldots, m\}$ such that $u \in B_{s}^{(l)}$. Let $X$ be an (ad 4)-pivot and $Y_{1} \rightarrow Y_{2} \rightarrow \cdots \rightarrow Y_{t}$ with $t \geq 1$, be a finite sectional path in $\Gamma_{A_{j}}$ (as in the definition of $\left.(\mathrm{ad} 4)\right)$. Note that this finite sectional path is the linearly oriented quiver of type $\mathbb{A}_{t}$ and its support algebra $\Lambda$ (given by the vertices corresponding to the simple composition factors of the modules $\left.Y_{1}, Y_{2}, \ldots, Y_{t}\right)$ is a tilted algebra of the path algebra $D$ of the linearly oriented quiver of type $\mathbb{A}_{t}$. From [34, (4.4)(2)] we know that $\Lambda$ is a bound quiver algebra given by a branch in $x$, where $x$ corresponds to the unique projectiveinjective $D$-module. Let $\Gamma$ be a generalized multicoil of $\Gamma_{A_{j+1}}$ obtained by applying the admissible operation ( $\operatorname{ad} 4)$, where $X$ is the pivot contained in the generalized multicoil $\Omega_{1}$, and $Y_{1}$ is the starting vertex of a finite sectional path contained in the generalized multicoil $\Omega_{1}$ or $\Omega_{2}$. So, $\Gamma$ is obtained from $\Omega_{1}$ or from the disjoin union of two generalized multicoils $\Omega_{1}, \Omega_{2}$ by the corresponding translation quiver admissible operations. Therefore, the bound quiver $Q_{A_{j+1}}$ of $A_{j+1}$ in the first case is of the form

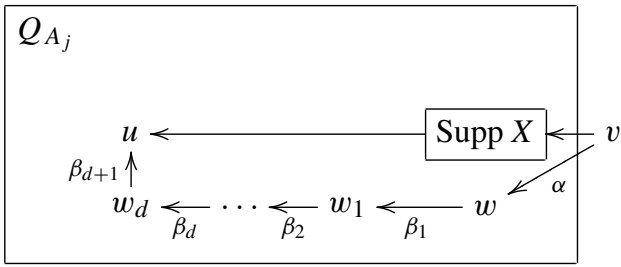


for $r=0$ and

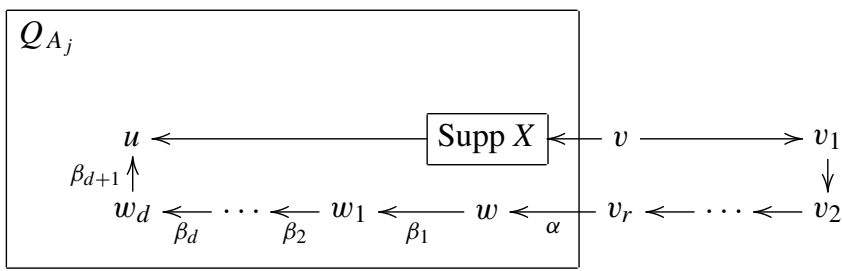

for $r \geq 1$, where the index $r$ is as in the definition of $(\operatorname{ad} 4), v$ is the extension point of $A_{j}[X], w$ is the extension point of $A_{j}\left[Y_{1}\right], w_{1}, \ldots, w_{d}$ belong to branch in $w$ generated by the support of $Y_{1} \oplus \cdots \oplus Y_{t}$, and $\alpha \beta_{1} \ldots \beta_{h}=0$ for some $h \in\{1, \ldots, d+1\}$. In the second case the bound quiver $Q_{A_{j+1}}$ of $A_{j+1}$ is of the form

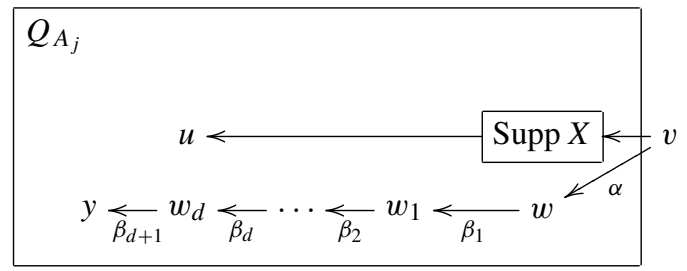

for $r=0$ and

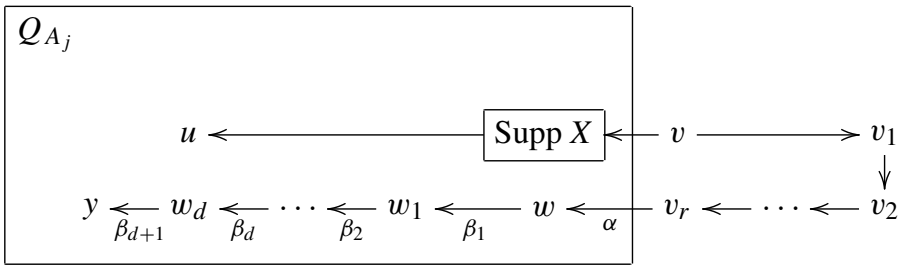

for $r \geq 1$, where the index $r$ is as in the definition of $(\operatorname{ad} 4), v$ is the extension point of $A_{j}[X], w$ is the extension point of $A_{j}\left[Y_{1}\right], w_{1}, \ldots, w_{d}$ belong to branch in $w$ generated by the support of $Y_{1} \oplus \cdots \oplus Y_{t}, \alpha \beta_{1} \ldots \beta_{h}=0$ for some $h \in\{1, \ldots, d+1\}$, and $y$ is the coextension point of $A_{j}$ such that $y \in A^{(l)} \backslash A^{(r)}$. More precisely, $y \in B_{s^{\prime}}^{(l)}$, where $s^{\prime} \in\{1, \ldots, m\}$ and $s^{\prime} \neq s$. Moreover in both cases, we have $P_{v}(u)=X(u)=k$ or $P_{v}(u)=X(u)=0$, and hence all non-zero paths from $v$ to $u$ are congruent modulo $I_{j+1}$. So, $A_{j+1}(v, u)$ is at most one-dimensional. We note that in the first case, the definition of (ad 4) (see the shape of the bound quiver $Q_{A_{j+1}}$ of $A_{j+1}$ ) implies that if the paths $\omega_{\alpha_{f}}, \omega_{\alpha_{g}} \in$ $A_{j+1}(v, u)$ are non-zero and $\omega_{\alpha_{f}}-\omega_{\alpha_{g}} \in I$, then there is also zero path $\omega_{\alpha_{h}} \in A_{j+1}(v, u)$ for some $h \in\{1, \ldots, q\}, h \neq f \neq g$.

Let $X$ be an (ad 5)-pivot and $\Gamma$ be a generalized multicoil of $\Gamma_{A_{j+1}}$ obtained by applying this admissible operation with pivot $X$. Then $\Gamma$ is obtained from the disjoint union of the finite family of generalized multicoils $\Omega_{1}, \Omega_{2}, \ldots, \Omega_{e}$ by the corresponding translation quiver admissible operations, $1 \leq e \leq l$, where $l$ is the number of stable tubes of $\Gamma_{C}$ used in the whole process of creating $\Gamma$. Since in the definition of admissible operation (ad 5) we use the finite versions (fad 1)- $(\mathrm{fad} 4)$ of the admissible operations $(\operatorname{ad} 1)-(\operatorname{ad} 4)$ and the admissible operation ( $\mathrm{ad}$ ), we conclude that the required statement follows from the above considerations. 


\subsection{The factor space branch}

Let $A$ be an algebra, $u$ be a coextension point of $A$, and $K$ be the branch with root vertex $u$. We denote the arrows in $K$ by $\alpha$ and $\beta$, where $\alpha \beta=0$. For a given vertex $w \in K$, which is the end point of an arrow $\beta$ (respectively, the starting point of an arrow $\alpha$ ), we denote by $\sigma(w)$ (respectively, $\tau(w)$ ) the vertex $v \in K$ for which the length of a path $v \stackrel{\beta}{\longrightarrow} \cdots \stackrel{\beta}{\longrightarrow} w$ (respectively, $w \stackrel{\alpha}{\longrightarrow} \cdots \stackrel{\alpha}{\longrightarrow} v$ ) is the largest possible. Following [34, (4.4)], we call the maximal subbranch of $K$ of the form $u \stackrel{\beta}{\longrightarrow} \cdots \stackrel{\beta}{\longrightarrow} y$ the factor space branch of $u$. Note that if $|\rightarrow v|=2$ and $v$ does not lie on the factor space branch od $u$, then $\tau(\sigma(v))$ is defined.

\subsection{Related pairs}

Let $A$ be a generalized multicoil enlargement of $C=C_{1} \times \cdots \times C_{m}, A^{(l)}=B_{1}^{(l)} \times \cdots \times B_{m}^{(l)}$ and $A^{(r)}=B_{1}^{(r)} \times \cdots \times B_{m}^{(r)}$, where $C_{1}, \ldots, C_{m}$ is a family of tame concealed algebras, and $B_{i}^{(l)}$ (respectively, $B_{i}^{(r)}$ ) is a unique maximal convex branch coextension (respectively, branch extension) of $C_{i}$ inside $A, i \in\{1, \ldots, m\}$. Moreover, let $C=A_{0}, \ldots, A_{p}=$ $A^{(l)}, A_{p+1}, \ldots, A_{n}=A$ be an admissible sequence for $A$. Then

(1) each operation of type $(\mathrm{ad} 2)$ or $(\mathrm{ad} 3)$ in the corresponding sequence of admissible operations yields a pair of vertices $(v, u)$ with $v \in A^{(r)} \backslash A^{(l)}$ and $u \in A^{(l)} \backslash A^{(r)}$ (see the proof of Lemma 4.3). More precisely, $v \in B_{s}^{(r)} \backslash B_{s}^{(l)}$ and $u \in B_{s}^{(l)} \backslash B_{s}^{(r)}$ for some $s \in\{1, \ldots, m\}$;

(2) we can apply the operations ( $\operatorname{ad} 4)$, (fad 4), (that is, also $(\operatorname{ad} 5)$ ) in two ways. The first way is when the sectional paths occurring in the definitions of these operations come from a component or two components of the same connected algebra. The second one is, when these sectional paths come from two components of two connected algebras. Only in the first case we can obtain a pair $(v, u)$ of vertices in $Q_{A}$ such that $v \in A^{(r)} \backslash A^{(l)}, u \in A^{(l)} \backslash A^{(r)}$ and for all arrows $\alpha_{1}, \ldots, \alpha_{q} \in v^{\rightarrow}, q \geq 2$ there are paths $\omega_{\alpha_{1}}, \ldots, \omega_{\alpha_{q}} \in A(v, u)$ (see the proof of Lemma 4.4).

Now, if we have two such pairs $\left(v_{1}, u_{1}\right)$ and $\left(v_{2}, u_{2}\right)$ of vertices obtained by applying operation of type $(\mathrm{ad} 2),(\mathrm{ad} 3),(\mathrm{ad} 4)$ or $(\mathrm{ad} 5)$ in the corresponding sequence of admissible operations, where $v_{1}, v_{2} \in A^{(r)} \backslash A^{(l)}$ and $u_{1}, u_{2} \in A^{(l)} \backslash A^{(r)}$, then in general, the following three cases are possible:

- the pairs $\left(v_{1}, u_{1}\right)$ and $\left(v_{2}, u_{2}\right)$ are obtained by applying the corresponding sequence of admissible operations with pivots belonging to the component of $\Gamma_{A}$ obtained from the same stable tube;

- the pairs $\left(v_{1}, u_{1}\right)$ and $\left(v_{2}, u_{2}\right)$ are obtained by applying the corresponding sequence of admissible operations with pivots belonging to the component of $\Gamma_{A}$ obtained from two different stable tubes of the same connected algebra;

- the pairs $\left(v_{1}, u_{1}\right)$ and $\left(v_{2}, u_{2}\right)$ are obtained by applying the corresponding sequence of admissible operations with pivots belonging to the component of $\Gamma_{A}$ obtained from two different stable tubes of two connected algebras.

If the first case occurs, then the pairs $\left(v_{1}, u_{1}\right)$ and $\left(v_{2}, u_{2}\right)$ we call related. It is easy to see that this is indeed an equivalence relation.

Lemma 4.7 Let $A$ be a generalized multicoil enlargement of $C=C_{1} \times \cdots \times C_{m}$ without exceptional configurations of modules, where $C_{1}, \ldots, C_{m}$ is a family of tame concealed 
algebras. Moreover, let $C=A_{0}, \ldots, A_{p}=A^{(l)}, A_{p+1}, \ldots, A_{n}=A$ be an admissible sequence for $A$, and $\left(v_{1}, u_{1}\right)$ and $\left(v_{2}, u_{2}\right)$ be two pairs of vertices obtained by applying operation of type $(\mathrm{ad} 2)$ or $(\mathrm{ad} 3)$ in the corresponding sequence of admissible operations, where $v_{1}, v_{2} \in A^{(r)} \backslash A^{(l)}$ and $u_{1}, u_{2} \in A^{(l)} \backslash A^{(r)}$. Then the following statements hold.

(i) If $\left(v_{1}, u_{1}\right),\left(v_{2}, u_{2}\right)$ are related pairs and $u_{2}$ does not lie on the factor space branch of a coextension vertex of $C$, then there must be a previous operation of type $(\operatorname{ad} 2)$ in the sequence for which the associated pair of vertices is $\left(v_{1}, \tau\left(\sigma\left(u_{2}\right)\right)\right)$.

(ii) If there is a path in $Q_{A}$ from $v_{2}$ to $v_{1}$, then $u_{1}$ and $u_{2}$ belong to the same coextension branch $K$ and there is a path $u_{2} \stackrel{\beta}{\longrightarrow} \cdots \stackrel{\beta}{\longrightarrow} u_{1}$ in $K$.

Proof By [27, Section 4], $A^{(l)}$ is a unique maximal convex branch coextension of $C=$ $C_{1} \times \cdots \times C_{m}$ inside $A$, that is, $A^{(l)}=B_{1}^{(l)} \times \cdots \times B_{m}^{(l)}$, where $B_{i}^{(l)}$ is a unique maximal convex branch coextension of $C_{i}$ inside $A, i \in\{1, \ldots, m\}$. More precisely, $B_{i}^{(l)}={ }_{j=1}^{t_{i}}\left[K_{j}\right.$, $\left.E_{j}\right] C_{i}$, where $K_{1}, \ldots, K_{t_{i}}$ are branches, $i \in\{1, \ldots, m\}$. Moreover, $A^{(r)}$ is a unique maximal convex branch extension of $C=C_{1} \times \cdots \times C_{m}$ inside $A$, that is, $A^{(r)}=B_{1}^{(r)} \times \cdots \times B_{m}^{(r)}$, where $B_{i}^{(r)}$ is a unique maximal convex branch extension of $C_{i}$ inside $A, i \in\{1, \ldots, m\}$.

(i) Let $\Gamma$ be the generalized multicoil of $\Gamma_{A}$ containing the pivot $X$ of the operation which yields $\left(v_{2}, u_{2}\right)$. Then $\Gamma$ contains a ray passing through $X$. On the other hand, in the tube of $\Gamma_{A^{(l)}}\left(=\Gamma_{B_{i}^{(l)}}\right.$ for some $\left.i \in\{1, \ldots, m\}\right)$, which is transformed into $\Gamma$ by the sequence of admissible operations, there is a sectional path from the indecomposable injective $A^{(l)}$. module $I_{u_{2}}$ to the simple $A^{(l)}$-module $S_{\sigma\left(u_{2}\right)}$, but there is no ray passing through both. In general, we can create such a ray by the application of an admissible operation of type ( $\operatorname{ad} 2),(\operatorname{ad} 4)$ or (ad 5). Since $\Gamma$ does not contain an exceptional configurations of modules, we get that only way to create that ray is by the application of an admissible operation of type $(\operatorname{ad} 2)$ with pivot the indecomposable injective $A^{(l)}$-module $I_{\tau \sigma\left(u_{2}\right)}$.

(ii) It follows from the discussion before the lemma, the assumption that there are no exceptional configurations of modules and from [14, Lemma 2.5 (b)].

We illustrate the above considerations in the following example.

Example 4.8 Let $A=k Q / I$ be the bound quiver algebra given by the quiver $Q$ of the form

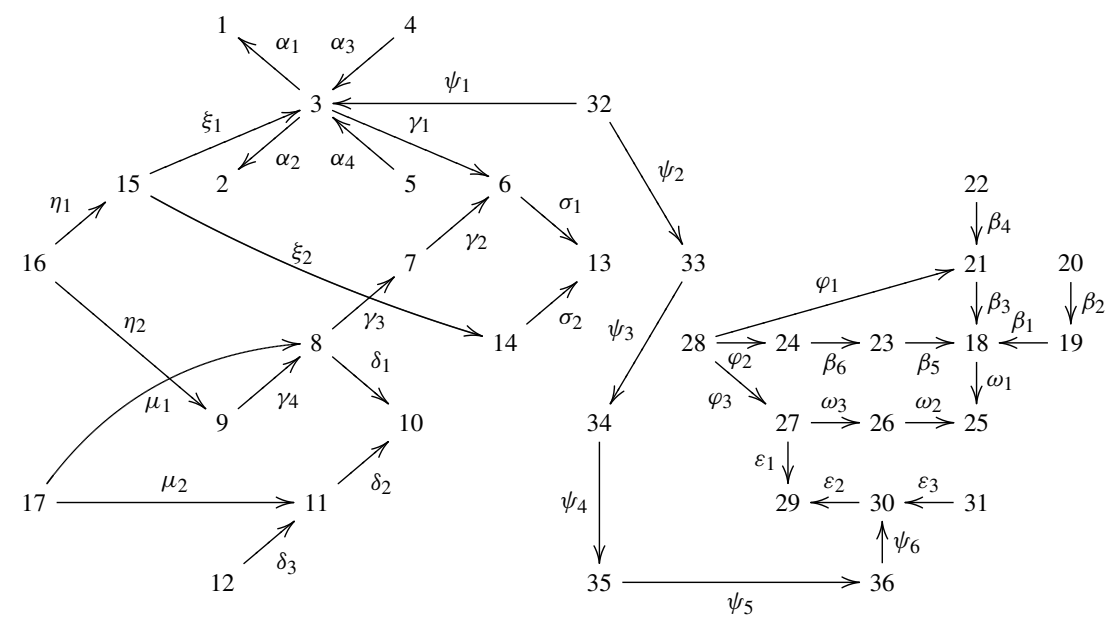


and $I$ the ideal in the path algebra $k Q$ of $Q$ over $k$ generated by the elements $\alpha_{3} \gamma_{1}, \alpha_{4} \gamma_{1}$, $\gamma_{4} \delta_{1}, \gamma_{2} \sigma_{1}, \xi_{1} \alpha_{1}, \xi_{1} \alpha_{2}, \xi_{1} \gamma_{1} \sigma_{1}-\xi_{2} \sigma_{2}, \eta_{1} \xi_{2}, \eta_{1} \xi_{1} \gamma_{1} \sigma_{1}, \eta_{1} \xi_{1} \gamma_{1}-\eta_{2} \gamma_{4} \gamma_{3} \gamma_{2}, \mu_{1} \gamma_{3}, \mu_{1} \delta_{1}-$ $\mu_{2} \delta_{2}, \beta_{4} \beta_{3} \omega_{1}, \beta_{1} \omega_{1}, \varphi_{1} \beta_{3}-\varphi_{2} \beta_{6} \beta_{5}, \varphi_{2} \beta_{6} \beta_{5} \omega_{1}-\varphi_{3} \omega_{3} \omega_{2}, \varphi_{3} \varepsilon_{1}, \psi_{1} \alpha_{1}, \psi_{1} \alpha_{2}, \psi_{6} \varepsilon_{2}$. We first show that $A$ is a generalized multicoil enlargement of $C=C_{1} \times C_{2}$, where $C_{1}$ and $C_{2}$ are tame concealed algebras. Indeed, let $C_{1}$ be the hereditary algebra of Euclidean type $\widetilde{\mathbb{D}}_{4}$ given by the vertices $1,2,3,4,5$, and $C_{2}$ be the hereditary algebra of Euclidean type $\mathbb{\mathbb { E }}_{6}$ given by the vertices $18,19,20,21,22,23,24$. Consider the dimension-vectors

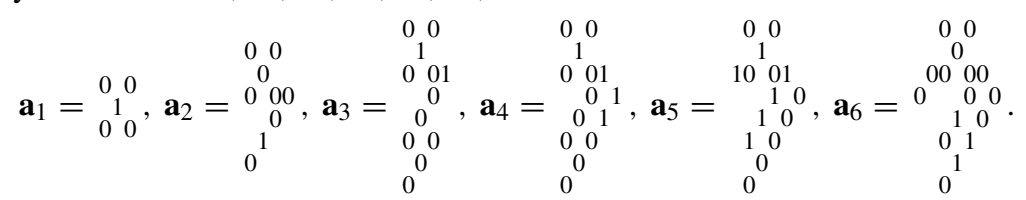

We apply (ad $\left.1^{*}\right)$ to $C_{1}$ with pivot the simple regular $C_{1}$-module with dimension-vector $\mathbf{a}_{1}$, and with parameter $t=3$. The modified algebra $A_{1}$ is given by the quiver with the vertices $1, \ldots, 9$ bound by $\alpha_{3} \gamma_{1}, \alpha_{4} \gamma_{1}$. Now, we apply (ad $1^{*}$ ) with pivot the indecomposable $A_{1}$-module with dimension-vector $\mathbf{a}_{2}$, and with parameter $t=2$. The modified algebra $A_{2}$ is given by the quiver with the vertices $1, \ldots, 12$ bound by $\alpha_{3} \gamma_{1}, \alpha_{4} \gamma_{1}, \gamma_{4} \delta_{1}$. Next, we apply ( $\left.\operatorname{ad} 1^{*}\right)$ with pivot the indecomposable $A_{2}$-module with dimension-vector $\mathbf{a}_{3}$, and with parameter $t=1$. The modified algebra $A_{3}$ is given by the quiver with the vertices $1, \ldots, 14$ bound by $\alpha_{3} \gamma_{1}, \alpha_{4} \gamma_{1}, \gamma_{4} \delta_{1}, \gamma_{2} \sigma_{1}$. In the next step we apply (ad 2) with pivot the indecomposable $A_{3}$-module with dimension-vector $\mathbf{a}_{4}$, and with parameter $t=1$. The modified algebra $A_{4}$ is given by the quiver with the vertices $1, \ldots, 15$ bound by $\alpha_{3} \gamma_{1}, \alpha_{4} \gamma_{1}, \gamma_{4} \delta_{1}$, $\gamma_{2} \sigma_{1}, \xi_{1} \alpha_{1}, \xi_{1} \alpha_{2}, \xi_{1} \gamma_{1} \sigma_{1}-\xi_{2} \sigma_{2}$. Now, we apply (ad 2) with pivot the indecomposable $A_{4^{-}}$ module with dimension-vector $\mathbf{a}_{5}$, and with parameter $t=3$. The modified algebra $A_{5}$ is given by the quiver with the vertices $1, \ldots, 16$ bound by $\alpha_{3} \gamma_{1}, \alpha_{4} \gamma_{1}, \gamma_{4} \delta_{1}, \gamma_{2} \sigma_{1}, \xi_{1} \alpha_{1}, \xi_{1} \alpha_{2}$, $\xi_{1} \gamma_{1} \sigma_{1}-\xi_{2} \sigma_{2}, \eta_{1} \xi_{2}, \eta_{1} \xi_{1} \gamma_{1} \sigma_{1}, \eta_{1} \xi_{1} \gamma_{1}-\eta_{2} \gamma_{4} \gamma_{3} \gamma_{2}$. Next, we apply (ad 3) with pivot the indecomposable $A_{5}$-module with dimension-vector $\mathbf{a}_{6}$, and with parameter $t=2$. The modified algebra $A_{6}$ is given by the quiver with the vertices $1, \ldots, 17$ bound by $\alpha_{3} \gamma_{1}, \alpha_{4} \gamma_{1}, \gamma_{4} \delta_{1}$, $\gamma_{2} \sigma_{1}, \xi_{1} \alpha_{1}, \xi_{1} \alpha_{2}, \xi_{1} \gamma_{1} \sigma_{1}-\xi_{2} \sigma_{2}, \eta_{1} \xi_{2}, \eta_{1} \xi_{1} \gamma_{1} \sigma_{1}, \eta_{1} \xi_{1} \gamma_{1}-\eta_{2} \gamma_{4} \gamma_{3} \gamma_{2}, \mu_{1} \gamma_{3}, \mu_{1} \delta_{1}-\mu_{2} \delta_{2}$.

Consider the algebra $C_{2}$. We will use the following dimension-vectors

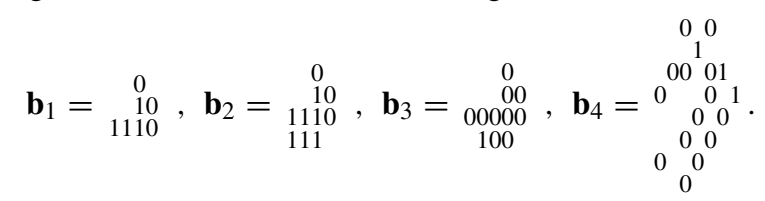

We apply $\left(\operatorname{ad~} 1^{*}\right)$ to $C_{2}$ with pivot the simple regular $C_{2}$-module with dimension-vector $\mathbf{b}_{1}$, and with parameter $t=2$. The modified algebra $B_{1}$ is given by the quiver with the vertices $18, \ldots, 27$ bound by $\beta_{1} \omega_{1}$. Now, we apply $(\operatorname{ad} 2)$ with pivot the indecomposable $B_{1}$-module with dimension-vector $\mathbf{b}_{2}$, and with parameter $t=2$. The modified algebra $B_{2}$ is given by the quiver with the vertices $18, \ldots, 28$ bound by $\beta_{1} \omega_{1}, \varphi_{1} \beta_{3}-\varphi_{2} \beta_{6} \beta_{5}, \varphi_{2} \beta_{6} \beta_{5} \omega_{1}-\varphi_{3} \omega_{3} \omega_{2}$. Next, we apply (ad $1^{*}$ ) with pivot the indecomposable $B_{2}$-module with dimension-vector $\mathbf{b}_{3}$, and with parameter $t=2$. The modified algebra $B_{3}$ is given by the quiver with the vertices $18, \ldots, 31$ bound by $\beta_{1} \omega_{1}, \varphi_{1} \beta_{3}-\varphi_{2} \beta_{6} \beta_{5}, \varphi_{2} \beta_{6} \beta_{5} \omega_{1}-\varphi_{3} \omega_{3} \omega_{2}, \varphi_{3} \varepsilon_{1}$. Finally, we apply (ad 4) with pivot the indecomposable $A_{6}$-module with dimension-vector $\mathbf{b}_{4}$, and with a finite sectional path consisting of the indecomposable $B_{3}$-modules with the following dimension-vectors

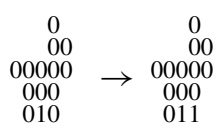


and with parameter $r=4$. The modified algebra is equal to $A$. Moreover, the left quasitilted algebra $A^{(l)}$ of $A$ is the convex subcategory of $A$ being the product $A^{(l)}=A_{1}^{(l)} \times A_{2}^{(l)}$, where $A_{1}^{(l)}=k Q_{1}^{(l)} / I_{1}^{(l)}$ is the branch coextension of the tame concealed algebra $C_{1}$ and $A_{2}^{(l)}=k Q_{2}^{(l)} / I_{2}^{(l)}$ is the branch coextension of the tame concealed algebra $C_{2}$ given by the quivers
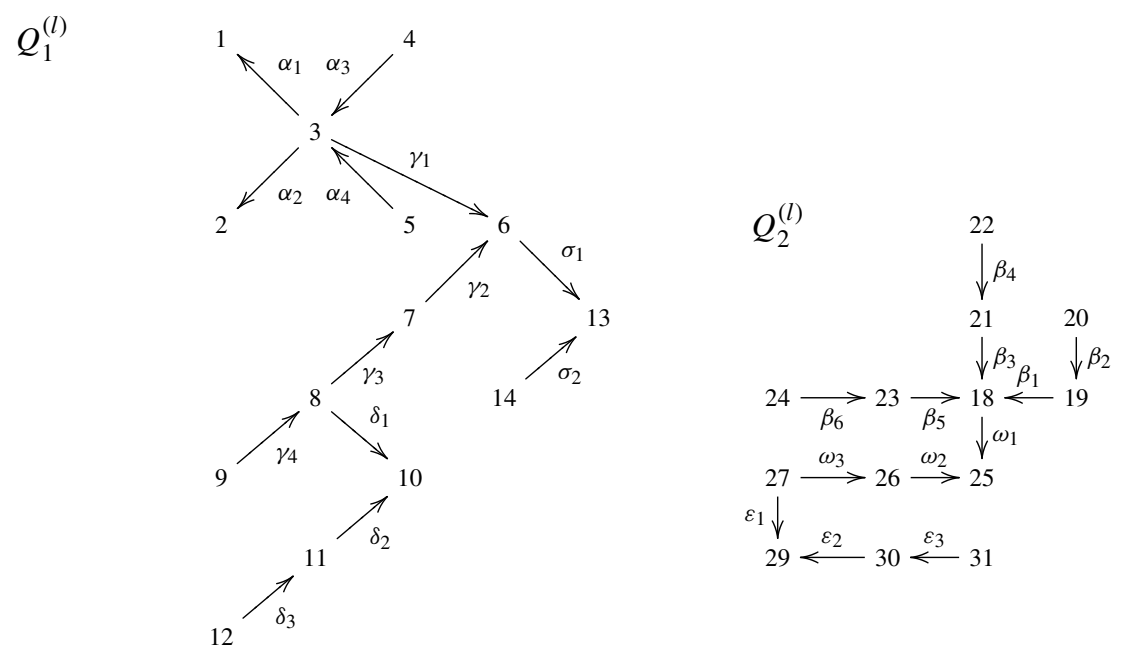

and the ideals $I_{1}^{(l)}=k Q_{1}^{(l)} \cap I$ in $k Q_{1}^{(l)}$ and $I_{2}^{(l)}=k Q_{2}^{(l)} \cap I$ in $k Q_{2}^{(l)}$. The right quasitilted algebra $A^{(r)}$ of $A$ is the convex subcategory of $A$ being the product $A^{(r)}=A_{1}^{(r)} \times A_{2}^{(r)}$, where $A_{1}^{(r)}=k Q_{1}^{(r)} / I_{1}^{(r)}$ is the branch coextension of the tame concealed algebra $C_{1}$ and $A_{2}^{(r)}=k Q_{2}^{(r)} / I_{2}^{(r)}$ is the branch coextension of the tame concealed algebra $C_{2}$ given by the quivers

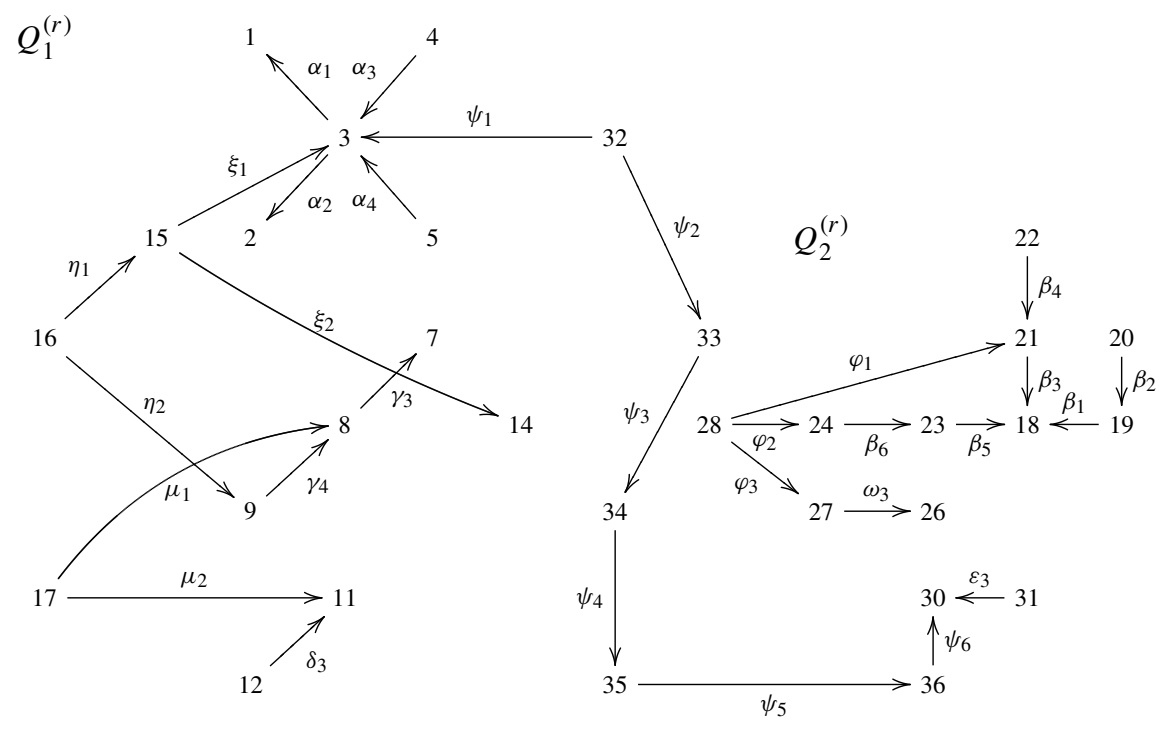

and the ideals $I_{1}^{(r)}=k Q_{1}^{(r)} \cap I$ in $k Q_{1}^{(r)}$ and $I_{2}^{(r)}=k Q_{2}^{(r)} \cap I$ in $k Q_{2}^{(r)}$. 
It follows from [27, Theorem A] and the above construction that the Auslander-Reiten quiver $\Gamma_{A}$ of the algebra $A=k Q / I$ admits a separating family of almost cyclic coherent components without exceptional configurations of modules.

Note that $(16,6)$ and $(17,10)$ are related pairs such that the vertex 10 does not lie on the factor space branch of a coextension vertex of $C=C_{1} \times C_{2}$ and $6=\tau(\sigma(10))$. Further, $(15,13)$ and $(16,6)$ are related pairs such that there is the path $16 \rightarrow 15$ in $Q$, and the vertices 13,6 belong to the same coextension branch and we have the path $6 \rightarrow 13$ in it. Moreover, the pairs $(28,25)$ and $(16,6)$ are not related nor do the pairs $(28,25),(17,10)$ and $(28,25),(15,13)$.

Corollary 4.9 Let A be a generalized multicoil enlargement of $C=C_{1} \times \cdots \times C_{m}$ without exceptional configurations of modules, where $C_{1}, \ldots, C_{m}$ is a family of tame concealed algebras. Moreover, let $C=A_{0}, \ldots, A_{p}=A^{(l)}, A_{p+1}, \ldots, A_{n}=A$ be an admissible sequence for $A$. The only vertices in $A^{(l)} \backslash A^{(r)}$ which can be sinks of a simple cycle in $Q_{A}$ are the coextension vertices of $C$ and the vertices $u$ that appear in a pair $(v, u)$ associated to an operation of type $(\mathrm{ad} 2)$ or $(\mathrm{ad} 3)$ in the corresponding sequence of admissible operations that leads from $A^{(l)}$ to $A$ and which lie on the factor space branch of a coextension vertex of $C$.

Proof Again, by [27, Section 4], $A^{(l)}$ is a unique maximal convex branch coextension of $C=C_{1} \times \cdots \times C_{m}$ inside $A$, that is, $A^{(l)}=B_{1}^{(l)} \times \cdots \times B_{m}^{(l)}$, where $B_{i}^{(l)}$ is a unique maximal convex branch coextension of $C_{i}$ inside $A, i \in\{1, \ldots, m\}$. More precisely, $B_{i}^{(l)}={ }_{j=1}^{t_{i}}$ $\left[K_{j}, E_{j}\right] C_{i}$, where $K_{1}, \ldots, K_{t_{i}}$ are branches, $i \in\{1, \ldots, m\}$. Moreover, $A^{(r)}$ is a unique maximal convex branch extension of $C=C_{1} \times \cdots \times C_{m}$ inside $A$, that is, $A^{(r)}=B_{1}^{(r)} \times$ $\cdots \times B_{m}^{(r)}$, where $B_{i}^{(r)}$ is a unique maximal convex branch extension of $C_{i}$ inside $A, i \in$ $\{1, \ldots, m\}$.

Let $u \in A^{(l)} \backslash A^{(r)}$ be a sink of a simple (reduced) cycle in $Q_{A}$. Then $u \in B_{s}^{(l)} \backslash B_{s}^{(r)}$ for some $s \in\{1, \ldots, m\}$. Therefore, $u$ lies on a coextension branch $K_{j}, j \in\left\{1, \ldots, t_{s}\right\}$ and $\left.\right|^{\rightarrow} u \mid=2$. Note that after applying admissible operation (ad 4) or (ad 5), we cannot obtain a simple cycle in $Q_{A}$ (see 4.6(2)), because by the assumption we do not have an exceptional configurations of modules in $\Gamma_{A}$. Now, the proof is identical as in [14, Corollarry 2.7].

\section{Proof of Theorem 1.1}

Let $A$ be an algebra with a separating family of almost cyclic coherent components in $\Gamma_{A}$ without exceptional configurations of modules. The necessity is obvious. To prove the sufficiency, observe first that by [27, Theorem $\mathrm{C}]$, there is a unique full convex subcategory $A^{(l)}=A_{1}^{(l)} \times \cdots \times A_{m}^{(l)}$ of $A$ which is a tubular coextension of a concealed canonical algebra $C=C_{1} \times \ldots \times C_{m}$ such that $A$ is obtained from $A^{(l)}$ by a sequence of admissible operations of types (ad 1)-(ad 5). We shall prove our claim by induction on the number of admissible operations leading from $A^{(l)}$ to the algebra $A$. Note that we can apply an admissible operation $(\operatorname{ad} 2),(\operatorname{ad} 3),(\operatorname{ad} 4)$ or $(\operatorname{ad} 5)$ if the number of all successors of the module $Y_{i}$ (which occurs in the definitions of the above admissible operations) is finite for each $1 \leq i \leq t$. Indeed, if it is not the case, then the family of generalized multicoils obtained after applying such admissible operation is not sincere, and then it is not separating. Let $C=A_{0}, \ldots, A_{p}=A^{(l)}, A_{p+1}, \ldots, A_{n}=A$ be an admissible sequence for $A$ and assume 
that $A_{p+1}=A$. In this case, if the admissible operation performed is $(\operatorname{ad} 1),(\operatorname{ad} 2)$ or $(\operatorname{ad} 3)$, then $m=1$. Moreover, since $\Gamma_{A}$ does not contain exceptional configuration of modules, we get that if the admissible operation performed is (ad 4) or (ad 5), then $m=2$. Let $v_{0}$ be the corresponding extension point. We note that $v_{0}$ is the root of a branch of $A^{(r)}$ if the operation performed is ( $\mathrm{ad} 1)$.

Assume that $A$ is not strongly simply connected. Then, by Theorem 2.6, the bound quiver $\left(Q_{A}, I\right)$ of $A$ contains an irreducible cycle which is not a contour, or an irreducible contour which is not naturally contractible in $\left(Q_{A}, I\right)$. As in [14, (3.1)] or [2, (4.4)], we consider two cases.

Case 1 Let $w=(p, q)$ be an irreducible contour from $v$ to $h$ which is not naturally contractible. Since $w$ lies neither inside $A^{(l)}$ nor inside $A^{(r)}$, and since $A^{(l)}$ is closed under successors and $A^{(r)}$ is closed under predecessors, we get that $v=v_{0}$ and $h \in A^{(l)} \backslash A^{(r)}$.

Let $\alpha_{1}: v \rightarrow a_{1}$ and $\alpha_{2}: v \rightarrow a_{2}$ be the starting arrows of $p$ and $q$, respectively. Since $v=v_{0}$ is separating, by [6, Section 2], $\alpha_{1}$ and $\alpha_{2}$ belong to the same equivalence class. We consider two subcases.

Subcase 1a Assume that there exists a minimal relation $\lambda_{1} \alpha_{1} x_{1}+\lambda_{2} \alpha_{2} x_{2}+\sum_{j \geq 3} \lambda_{j} y_{j}$ (see [6, (2.4)]) from $v$ to a vertex $u \in A^{(l)}$ involving $\alpha_{1}$ and $\alpha_{2}$, where $0 \neq \lambda_{i} \in k$. If the admissible operation performed is (ad 1), then $u \in C$. Since $h$ does not belong to $A^{(r)}$, it follows that $u \neq h$ and there is no path from $h$ to $u$. Moreover, there is no path from $u$ to $h$ because $w$ is irreducible. If the admissible operation performed is (ad 2) or (ad 3), we can take $u$ as the vertex in $A^{(l)} \backslash A^{(r)}$ described in Lemma 4.3. Since $w$ is not naturally contractible, it follows that $u \neq h$ and there is no path from $h$ to $u$. Also, there is no path from $u$ to $h$ because $w$ is irreducible. Assume that the admissible operation performed is $(\mathrm{ad} 4)$ or $(\mathrm{ad} 5)$. Since, by the assumption, the sectional paths occurring in the definitions of the operations ( $\mathrm{ad} 4)$, (fad 4) come from two components of two connected algebras, we infer that $u \in C$ or $u \in A^{(l)} \cap A^{(r)} \backslash C$. Then in both cases, since $h \notin A^{(r)}$, we get that $u \neq h$ and there is no path from $h$ to $u$. Also, there is no path from $u$ to $h$ because $w$ is irreducible.

Let $b_{1}$ (or $b_{2}$ ) be the last common vertex between $x_{1}$ and $p$ (or $x_{2}$ and $q$, respectively) and $u^{\prime}$ be the first common vertex between $x_{1}$ and $x_{2}$. Denote by $x_{1}^{\prime}$ (or $x_{2}^{\prime}$ ) the subpath of $x_{1}$ (or $x_{2}$ ) from $b_{1}$ (or $b_{2}$, respectively) to $u^{\prime}$, and by $p^{\prime}$ (or $q^{\prime}$ ) the subpath of $p$ (or $q$ ) from $b_{1}$ (or $b_{2}$ ) to $h$.

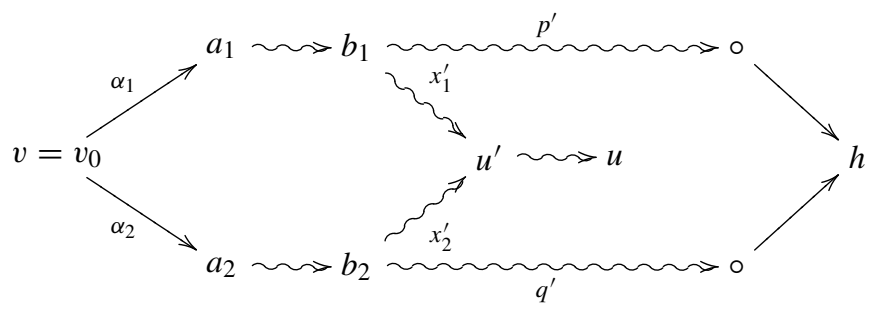

Consider now the walk $w^{\prime}=x_{2}^{\prime-1} q^{\prime} p^{\prime-1} x_{1}^{\prime}$. Note that this walk is a cycle. Indeed, there is no intersection between $p^{\prime}$ and $q^{\prime}$, nor is there one between $x_{1}^{\prime}$ and $x_{2}^{\prime}$, and the existence of an intersection between $x_{2}^{\prime} x_{1}^{\prime-1}$ and $p^{\prime} q^{\prime-1}$ would contradict the irreducibility of $w$. Moreover, $w^{\prime}$ is irreducible because $w$ is. Finally, $w^{\prime}$ is not a contour because it has two different sinks $u^{\prime}$ and $h$. Since $w^{\prime}$ lies inside $A^{(l)}$, we obtain a contradiction. 
Subcase 1b Assume now that does not exist a minimal relation involving $\alpha_{1}$ and $\alpha_{2}$. Since the equivalent class of an arrow starting at $v$ is the smallest equivalent relation, we have that $\alpha_{1}$ and $\alpha_{2}$ belong to the same equivalent class if there exists a sequence of arrows $\beta_{1}, \ldots, \beta_{s}$, $s \geq 1$ such that there exists a sequence of minimal relations $\rho_{1}, \ldots, \rho_{s+1}$ satisfying the following sequence of conditions: $\alpha_{1}$ and $\beta_{1}$ are arrows in $\rho_{1}, \beta_{2}$ and $\beta_{3}$ are arrows in $\rho_{2}$, $\ldots, \beta_{s}$ and $\alpha_{2}$ are arrows in $\rho_{s+1}$. Using this sequence of minimal relations and the same arguments as in the previous subcase, we will get an analogous cycle and a corresponding contradiction.

Case 2 Let $w$ be an irreducible cycle which is not a contour. Denote by $v_{1}, \ldots, v_{t}$ its sources, by $h_{1}, \ldots, h_{t}$ its sinks and for $1 \leq i \leq t$, by $w_{i}$ the path from $v_{i}$ to $h_{i}$, and by $w_{i}^{\prime}$ the path from $v_{i}$ to $h_{i+1}$ (where $h_{t+1}=h_{1}$ ). Since $w$ lies neither inside $A^{(l)}$ nor inside $A^{(r)}$, we may assume that $v_{1}=v_{0}$ and $h_{s} \in A^{(l)} \backslash A^{(r)}$, where $1 \leq s \leq t$. Let $\alpha_{1}: v_{1} \rightarrow a_{1}$ and $\alpha_{2}: v_{1} \rightarrow a_{2}$ be the starting arrows of $w_{1}$ and $w_{1}^{\prime}$, respectively. Since $v_{1}$ is separating, applying [6, Section 2], we conclude that $\alpha_{1}$ and $\alpha_{2}$ belong to the same equivalence class. As before, we consider two subcases.

Subcase 2a Assume that there exists a minimal relation $\lambda_{1} \alpha_{1} x_{1}+\lambda_{2} \alpha_{2} x_{2}+\sum_{j \geq 3} \lambda_{j} y_{j}$ (see [6, (2.4)]) from $v_{1}$ to a vertex $u \in A^{(l)}$ involving $\alpha_{1}$ and $\alpha_{2}$, where $0 \neq \lambda_{i} \in k$. If the admissible operation performed is ( $\operatorname{ad} 1)$, then $u \in C$ and $u \neq h_{s}$ and there is no path from $h_{s}$ to $u$. Also, by the irreducibility of $w$, there is no path from $u$ to $h_{s}$. If the admissible operation performed is (ad 2) or (ad 3), then $u$ can be taken as the vertex in $A^{(l)} \backslash A^{(r)}$ described in Lemma 4.3. Since $w$ is irreducible, it follows that $u \neq h_{s}$, there is no path from from $u$ to $h_{s}$ and none from $h_{s}$ to $u$ (otherwise, $h_{l}$ is the root of the branch in which $u$ lies and there is a path from $v_{1}$ to $h_{s}$ that reduces the cycle). If the admissible operation performed is $(\mathrm{ad} 4)$ or ( $\mathrm{ad} \mathrm{5)} \mathrm{then,} \mathrm{by} \mathrm{the} \mathrm{assumption,} \mathrm{the} \mathrm{sectional} \mathrm{paths} \mathrm{occurring} \mathrm{in} \mathrm{the}$ definitions of the operations ( $\mathrm{ad} 4)$, (fad 4) come from two components of two connected algebras, we get that $u \in C$ or $u \in A^{(l)} \cap A^{(r)} \backslash C$. Then in both cases, since $h_{s} \notin A^{(r)}$, we obtain that $u \neq h_{s}$ and there is no path from $h_{s}$ to $u$. Again, by the irreducibility of $w$, we have that there is no path from $u$ to $h_{s}$.

Let $b_{1}$ (or $b_{2}$ ) be the last common vertex of $x_{1}$ (or $x_{2}$ ) and $w_{1}$ (or $w_{1}^{\prime}$, respectively), and $u^{\prime}$ be the first common vertex of $x_{1}$ and $x_{2}$. Denote by $x_{1}^{\prime}$ (or $x_{2}^{\prime}$ ) the subpath of $x_{1}$ (or $x_{2}$ ) from $b_{1}$ (or $b_{2}$, respectively) to $u^{\prime}$, and by $w_{1}^{\prime \prime}$ (or $w_{2}^{\prime \prime}$ ) the subpath of $w_{1}$ (or $w_{1}^{\prime}$ ) from $b_{1}$ (or $b_{2}$ ) to $h_{1}$ (or $h_{2}$, respectively).

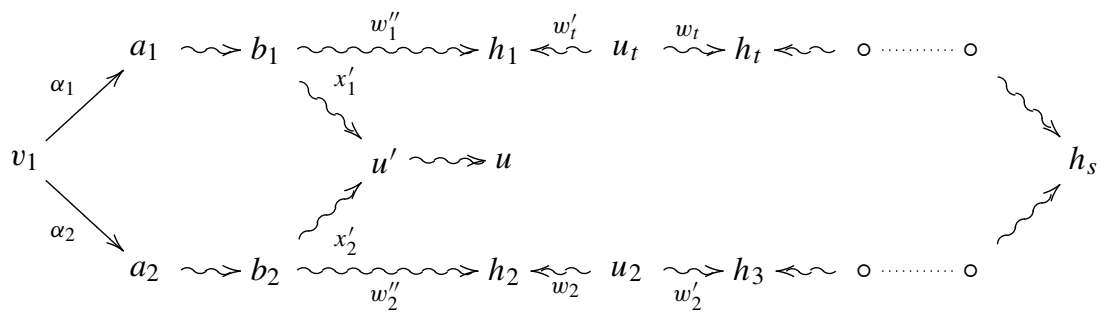

Note that either $t \geq 3$ or, if $t=2$, then $b_{1} \neq h_{1}$ or $b_{2} \neq h_{2}$. Indeed, if $t=2$, then one of $h_{1}$ or $h_{2}$ is equal to $h_{s}$ and we have just shown that there is no path from $h_{s}$ to $u$. Consider now the walk $w^{\prime}=w_{1}^{\prime \prime} w_{t}^{\prime-1} w_{t} \ldots w_{2} w_{2}^{\prime \prime} x_{2}^{\prime} x_{1}^{\prime-1}$. This walk is a cycle. Indeed, the walk $w_{1}^{\prime \prime} w_{t}^{\prime-1} w_{t} \ldots w_{2} w_{2}^{\prime \prime-1}$ has no self-intersection because it is a subwalk of $w$, the 
walk $x_{2}^{\prime} x_{1}^{\prime-1}$ has no self-intersection by definition, and these two do not intersect because $w$ is irreducible. Moreover, $w^{\prime}$ is irreducible because $w$ is. Finally, it is not a contour because it has at least two sinks $\left(u^{\prime}\right.$ and $\left.h_{s}\right)$. Since $w^{\prime}$ lies inside $A^{(l)}$, we get a contradiction.

Subcase 2b Assume now that does not exist a minimal relation involving $\alpha_{1}$ and $\alpha_{2}$. Since the equivalent class of an arrow starting at $v_{1}$ is the smallest equivalent relation, we have that $\alpha_{1}$ and $\alpha_{2}$ belong to the same equivalent class if there exists a sequence of arrows $\beta_{1}, \ldots, \beta_{s}$, $s \geq 1$ such that there exists a sequence of minimal relations $\rho_{1}, \ldots, \rho_{s+1}$ satisfying the following sequence of conditions: $\alpha_{1}$ and $\beta_{1}$ are arrows in $\rho_{1}, \beta_{2}$ and $\beta_{3}$ are arrows in $\rho_{2}$, $\ldots, \beta_{s}$ and $\alpha_{2}$ are arrows in $\rho_{s+1}$. Using this sequence of minimal relations and the same arguments as in the previous subcase, we will get an analogous cycle and a corresponding contradiction.

The contradictions obtained in both cases show that $A_{p+1}$ is strongly simply connected.

Let $k>p, A=A_{k+1}$ and assume that $A_{k}$ is strongly simply connected, but $A$ is not. Let $v_{0}$ be the extension point of $A_{k}$ and $X \in$ ind $A_{k}$ be the pivot of the admissible operation. If $X$ is the pivot of the admissible operation (ad 1), then $\left.X\right|_{C_{i}} \neq 0$ for some $i \in\{1, \ldots, m\}$. Indeed, otherwise, by Lemma 4.2(ii), $X$ would be uniserial, and since $A$ is obtained from $A_{k}$ by the one-point extension by $X$ followed by several one-point coextensions by simple modules, by [4, Theorem 3.4], A would be strongly simply connected, contrary to our assumption.

As before, by Theorem 2.6, the bound quiver $\left(Q_{A}, I\right)$ of $A$ contains an irreducible cycle which is not a contour, or an irreducible contour which is not naturally contractible in $\left(Q_{A}, I\right)$.

Case 1 Let $w=(p, q)$ be an irreducible contour from $v$ to $h$ which is not naturally contractible. Since $w$ lies neither inside $A_{k}$ nor inside $A^{(r)}$, we deduce that $v=v_{0}$ and $h \in A^{(l)} \backslash A^{(r)}$. Now, we can then proceed as in the first step of induction to obtain an irreducible cycle which is not a contour lying inside $A_{k}$, thus contradicting our assumption.

Case 2 Let $w$ be an irreducible cycle which is not a contour. Denote by $v_{1}, \ldots, v_{t}$ its sources, by $h_{1}, \ldots, h_{t}$ its sinks and for $1 \leq i \leq t$, by $w_{i}$ the path from $v_{i}$ to $h_{i}$, and by $w_{i}^{\prime}$ the path from $v_{i}$ to $h_{i+1}$ (where $h_{t+1}=h_{1}$ ). As above, we may assume that $v_{1}=v_{0}$ and $h_{s} \in A^{(l)} \backslash A^{(r)}$, where $1 \leq s \leq t$. Moreover, let $\alpha_{1}: v_{1} \rightarrow a_{1}$ and $\alpha_{2}: v_{1} \rightarrow a_{2}$ be the starting arrows of $w_{1}$ and $w_{1}^{\prime}$, respectively. Since $v_{1}$ is separating, we have that $\alpha_{1}$ and $\alpha_{2}$ belong to the same equivalence class. As before, we consider two subcases.

Assume first that there exists a minimal relation $\lambda_{1} \alpha_{1} x_{1}+\lambda_{2} \alpha_{2} x_{2}+\sum_{j \geq 3} \lambda_{j} y_{j}$ from $v_{1}$ to a vertex $u \in A_{k}$ involving $\alpha_{1}$ and $\alpha_{2}$, where $0 \neq \lambda_{i} \in k$. If the admissible operation applied is (ad 1), then $u \in C$ (more precisely, $u \in C_{i}$ fore some $i \in\{1, \ldots, m\}$ ) and we can proceed as in the first step of induction. If the admissible operation applied is (ad 2) or (ad 3), then $u$ is the vertex in $A^{(l)} \backslash A^{(r)}$ described in Lemma 4.3. Again the irreducibility of $w$ gives that $u \neq h_{s}$, there is no path from from $u$ to $h_{s}$ and none from $h_{s}$ to $u$ in case $h_{s}$ is a coextension point of $C$. If $h_{s}$ is not a coextension point of $C$, then by Corollary 4.9 there is no path from $h_{s}$ to $u$. Indeed, otherwise $v_{0}$ is not constructible since $\mathcal{S}(X)$ does not have the proper shape. If the admissible operation applied is $(\mathrm{ad} 4)$ or $(\mathrm{ad} 5)$ then, by the assumption, the sectional paths occurring in the definitions of the operations ( $\mathrm{ad} 4)$, ( $\mathrm{fad} 4)$ come from two components of two connected algebras, we get that $u \in C_{i}$ or $u \in A_{i}^{(l)} \cap A_{i}^{(r)} \backslash C_{i}$ fore 
some $i \in\{1, \ldots, m\}$. Then in both cases, since $h_{s} \notin A_{i}^{(r)}$, we obtain that $u \neq h_{s}$ and there is no path from $h_{s}$ to $u$. Again, by the irreducibility of $w$, we have that there is no path from $u$ to $h_{s}$. Now, we can proceed as in the subcase 2a in the first step of induction to obtain an irreducible cycle which is not a contour lying inside $A_{k}$, contrary to our assumption.

Assume now that does not exist a minimal relation involving $\alpha_{1}$ and $\alpha_{2}$. We can proceed as in the subcase $2 b$ in the first step of induction to obtain a corresponding contradiction.

Finally, the contradictions obtained in both cases show that $A_{k+1}$ is strongly simply connected. This finishes the proof of the theorem.

\section{Proof of Theorem 1.2}

Let $A$ be a tame algebra with a separating family of almost cyclic coherent components in $\Gamma_{A}$ without exceptional configurations of modules. Then, by [27, Theorem $\mathrm{C}$ ], there are the left quasitilted quotient algebra $A^{(l)}$ of $A$ and the right quasitilted quotient algebra $A^{(r)}$ of $A$. Moreover, $A$ is tame if and only if $A^{(l)}$ and $A^{(r)}$ are tame. Then, it follows from [27, Theorem F] that $A^{(l)}$ and $A^{(r)}$ are products of tilted algebras of Euclidean type or tubular algebras. Therefore, the equivalence (ii), (iv) and (v) follows from [3, Corollary 2.3] and [1, Theorem 1.6]. By Theorem 1.1, (i) and (ii) are equivalent. Clearly, (i) implies (iii), and (iii) implies (iv). Since, by [38, Theorem 4.1], (i) implies (iv), it remains to show that (vi) implies (ii).

We know from [27, Theorem A] that the Auslander-Reiten quiver $\Gamma_{A}$ of $A$ admits a separating family of almost cyclic coherent components if and only if $A$ is a generalized multicoil enlargement of a finite family of concealed canonical algebras. But in the tame case we may restrict to the generalized multicoil enlargements of tame concealed algebras. Namely, we have the following consequence of [27, Theorems $\mathrm{A}$ and $\mathrm{F}]: A$ is tame and $\Gamma_{A}$ admits a separating family of almost cyclic coherent components if and only if $A$ is a tame generalized multicoil enlargement of a finite family $C_{1}, \ldots, C_{m}$ of tame concealed algebras. Let $A^{(l)}=A_{1}^{(l)} \times \cdots \times A_{m}^{(l)}$ and $A^{(r)}=A_{1}^{(r)} \times \cdots \times A_{m}^{(r)}$, then

- for each $i \in\{1, \ldots, m\}, A_{i}^{(l)}$ is a tubular coextension of the tame concealed algebra $C_{i}$ of Euclidean or tubular type;

- for each $i \in\{1, \ldots, m\}, A_{i}^{(r)}$ is a tubular extension of the tame concealed algebra $C_{i}$ of Euclidean or tubular type.

Now, if $A_{i}^{(l)}$ (respectively, $A_{i}^{(r)}$ ), $i \in\{1, \ldots, m\}$, is tubular, the separation condition for $A_{i}^{(l)}$ and $\left(A_{i}^{(l)}\right)^{\mathrm{op}}$ (respectively, $A_{i}^{(r)}$ and $\left(A_{i}^{(r)}\right)^{\mathrm{op}}$ ) implies that $A_{i}^{(l)}$ (respectively, $A_{i}^{(r)}$ ) is strongly simply connected according to [1, Theorem 1.6]. If $A_{i}^{(l)}$ (respectively, $A_{i}^{(r)}$ ), $i \in$ $\{1, \ldots, m\}$, is domestic, the separation condition for $\left(A_{i}^{(l)}\right)^{\text {op }}$ (respectively, $\left.A_{i}^{(r)}\right)$ implies that $A_{i}^{(l)}$ (respectively, $A_{i}^{(r)}$ ) does not contain a full convex subcategory which is hereditary of type $\widetilde{\mathbb{A}}_{n}$ according to [1, Lemma 1.5]. Then, by [3, Corollary 2.3], $A_{i}^{(l)}$ (respectively, $\left.A_{i}^{(r)}\right)$ is strongly simply connected.

\section{Proof of Corollary 1.3}

Let $A$ be an algebra. We know from [40, Theorem 4.1] that $A$ is cycle-finite and $\Gamma_{A}$ admits a sincere stable tube if and only if $A$ is either tame concealed or tubular algebra. Applying 
now the description of the structure of the Auslander-Reiten quiver of a tame generalized multicoil algebra [25, Theorem 4.8] (that is, a tame generalized multicoil enlargement of a finite family of tame concealed algebras), we conclude that any such algebra is cycle-finite. Using additionally [27, Theorem $\mathrm{A}]$ in the tame case, we receive that $A$ is tame and $\Gamma_{A}$ admits a separating family of almost cyclic coherent components if and only if $A$ is cyclefinite and $\Gamma_{A}$ admits a separating family of almost cyclic coherent components. Now, the Corollary 1.3 is a direct consequence of Theorem 1.2.

\section{Examples}

Example 8.1 Let $A=k Q / I$ be the bound quiver algebra given by the quiver $Q$ of the form

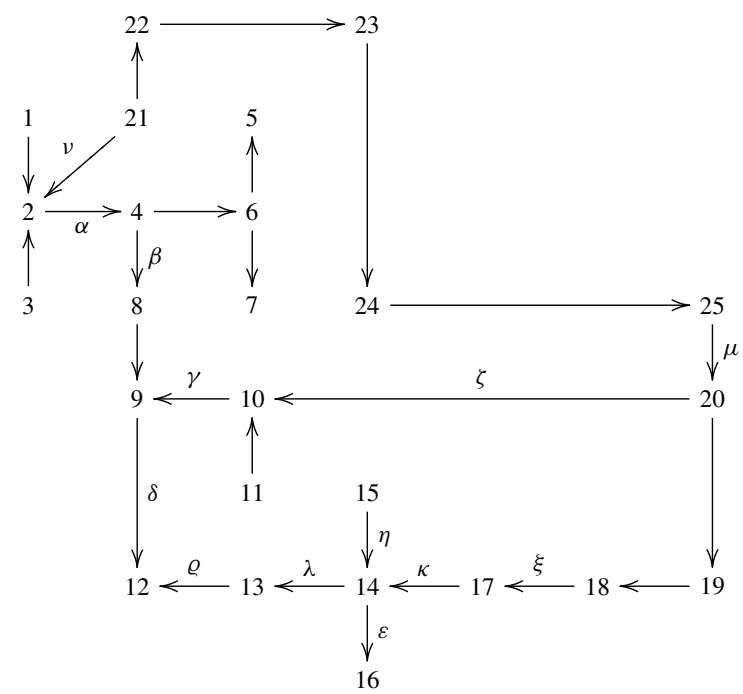

and $I$ the ideal in the path algebra $k Q$ of $Q$ over $k$ generated by the elements $\alpha \beta, \gamma \delta$, $\eta \varepsilon, \kappa \lambda \varrho, \zeta \gamma, \xi \kappa \lambda, \mu \zeta, v \alpha$. Then $A$ is a generalized multicoil enlargement of a concealed canonical algebra $C$, where $C$ is the hereditary algebra of Euclidean type $\widetilde{\mathbb{D}}_{6}$ given by the vertices $1,2, \ldots, 7$. Indeed, consider the dimension-vectors

$$
\begin{aligned}
& \mathbf{a}_{1}=\underset{010}{010}, \mathbf{a}_{2}=\begin{array}{c}
00 \\
010
\end{array}, \mathbf{a}_{3}=\begin{array}{c}
00 \\
010 \\
010 \\
10 \\
0
\end{array}, \mathbf{a}_{4}=\begin{array}{c}
00 \\
000 \\
000 \\
00 \\
00 \\
001
\end{array}, \\
& \mathbf{b}_{1}=\begin{array}{cc}
00 \\
000 \\
000 \\
00 \\
00 \\
011 \\
1
\end{array}, \mathbf{b}_{2}=\begin{array}{c}
00 \\
000 \\
000 \\
01 \\
00 \\
0000 \\
0
\end{array}, \mathbf{b}_{3}=\begin{array}{c}
000 \\
000 \\
00 \\
000000 \\
0
\end{array} \quad 0
\end{aligned}
$$

We apply (ad $\left.1^{*}\right)$ with pivot the simple regular $C$-module with vector $\mathbf{a}_{1}$, and with parameter $t=0$. The modified algebra $B_{1}$ is given by the quiver with vertices $1,2, \ldots, 8$ bound by $\alpha \beta=0$. Now, we apply (ad $\left.1^{*}\right)$ with pivot the indecomposable $B_{1}$-module with vector 
$\mathbf{a}_{2}$, and with parameter $t=2$. The modified algebra $B_{2}$ is given by the quiver with vertices $1,2, \ldots, 11$ bound by $\alpha \beta=0$. Next, we apply (ad $1^{*}$ ) with pivot the indecomposable $B_{2}$-module with vector $\mathbf{a}_{3}$, and with parameter $t=3$. The modified algebra $B_{3}$ is given by the quiver with vertices $1,2, \ldots, 15$ bound by $\alpha \beta=0, \gamma \delta=0$. In the next step we apply (ad $\left.1^{*}\right)$ with pivot the indecomposable $B_{3}$-module with vector $\mathbf{a}_{4}$, and with parameter $t=0$. The modified algebra $B_{4}$ is given by the quiver with vertices $1,2, \ldots, 16$ bound by $\alpha \beta=0, \gamma \delta=0, \eta \varepsilon=0$. Next, we apply the admissible operation (ad 5) in three steps. The first step: we apply the operation (fad 3 ) with pivot the indecomposable $B_{4}$-module with vector $\mathbf{b}_{1}$, and with parameters $t=3, s=2$. The modified algebra $B_{5}$ is given by the quiver with vertices $1,2, \ldots, 17$ bound by $\alpha \beta=0, \gamma \delta=0, \eta \varepsilon=0, \kappa \lambda \varrho=0$. The second step: we apply the operation (fad 4 ) with pivot the indecomposable $B_{5}$-module with vector $\mathbf{b}_{2}$, and with a finite sectional path consisting of the indecomposable $B_{5}$-modules with dimension-vectors

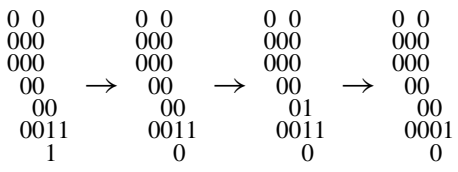

and with parameters $s=1, r=2$. The modified algebra $B_{6}$ is given by the quiver with vertices $1,2, \ldots, 20$ bound by $\alpha \beta=0, \gamma \delta=0, \eta \varepsilon=0, \kappa \lambda \varrho=0, \zeta \gamma=0, \xi \kappa \lambda=0$. The third step: we apply the operation (ad 4) with pivot the indecomposable $B_{6}$-module with vector $\mathbf{b}_{3}$, and with a finite sectional path consisting of the indecomposable $B_{6}$-modules with dimension-vectors

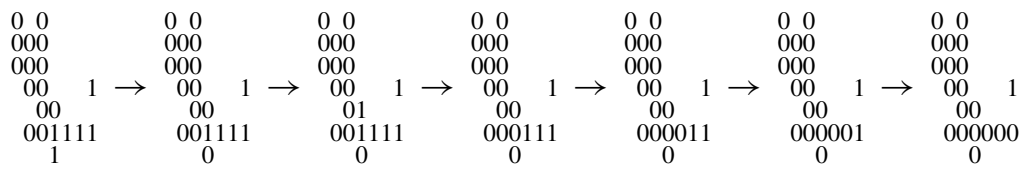

and with parameter $r=4$. The modified algebra is equal to $A$. Then the left quasitilted algebra $A^{(l)}$ of $A$ is the convex subcategory of $A$ being the bound quiver algebra $k Q^{(l)} / I^{(l)}$, where $Q^{(l)}$ is a full subquiver of $Q$ given by the vertices $1,2, \ldots, 16$ and $I^{(l)}=k Q^{(l)} \cap I$ is the ideal in $k Q^{(l)}$. The right quasitilted algebra $A^{(r)}$ of $A$ is the convex subcategory of $A$ being the bound quiver algebra $k Q^{(r)} / I^{(r)}$, where $Q^{(r)}$ is a full subquiver of $Q$ given by the vertices $1,2, \ldots, 7,14,15, \ldots, 25$ and $I^{(r)}=k Q^{(r)} \cap I$ is the ideal in $k Q^{(r)}$.

It follows from [27, Theorem A] and the above construction that the Auslander-Reiten quiver $\Gamma_{A}$ of the tame algebra $A=k Q / I$ admits a separating family of almost cyclic coherent components with exceptional configurations of modules. Moreover, by [30, Theorem 1.1], the first Hochschild cohomology space $H^{1}(A) \cong k^{2}$, and so $A$ is not strongly simply connected. We also note that, since $A^{(l)}$ and $A^{(r)}$ are tame tilted algebras of Euclidean type $\widetilde{\mathbb{D}}$ such that $H^{1}\left(A^{(l)}\right)=0$ and $H^{1}\left(A^{(r)}\right)=0$, it follows from [5, Theorem] that $A^{(l)}$ and $A^{(r)}$ are strongly simply connected. We refer to [27, Example 4.1] (see also [29, Example 9.13]) for the example of the tame algebra with a separating family of almost cyclic coherent components which is not strongly simply connected, because contains a full convex subcategory which is hereditary of type $\widetilde{\mathbb{A}}_{5}$. 
Example 8.2 Let $A=k Q / I$ be the bound quiver algebra given by the quiver $Q$ of the form

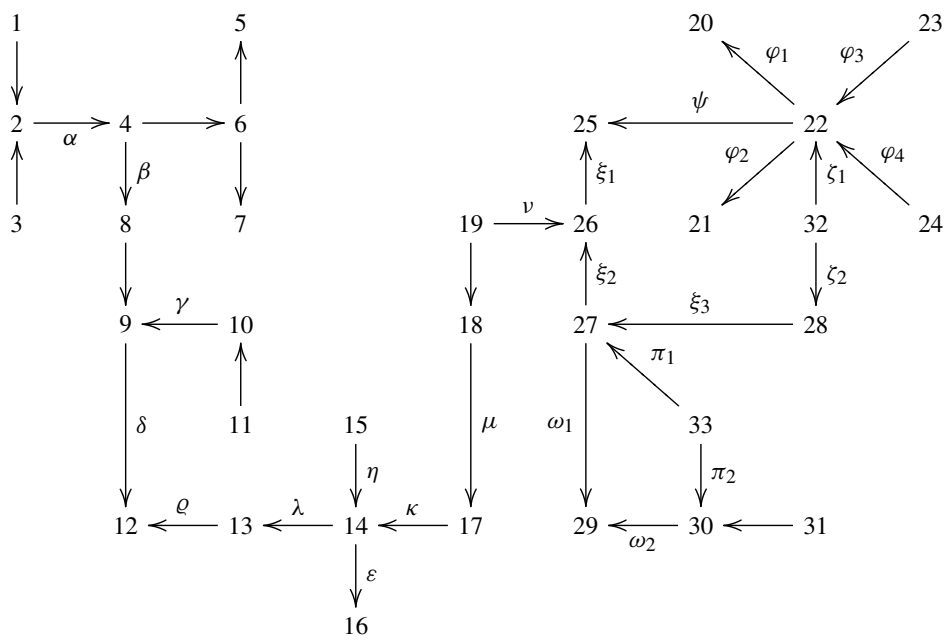

and $I$ the ideal in the path algebra $k Q$ of $Q$ over $k$ generated by the elements $\alpha \beta, \gamma \delta, \eta \varepsilon$, $\kappa \lambda \varrho, \varphi_{3} \psi, \varphi_{4} \psi, \xi_{3} \omega_{1}, \zeta_{1} \varphi_{1}, \zeta_{1} \varphi_{2}, \zeta_{2} \xi_{3} \xi_{2} \xi_{1}-\zeta_{1} \psi, \pi_{1} \xi_{2}, \pi_{1} \omega_{1}-\pi_{2} \omega_{2}, \mu \kappa \lambda, \nu \xi_{1}$. Then $A$ is a generalized multicoil enlargement of a concealed canonical algebra $C=C_{1} \times C_{2}$, where $C_{1}$ is the hereditary algebra of Euclidean type $\widetilde{\mathbb{D}}_{6}$ given by the vertices $1,2, \ldots, 7$, and $C_{2}$ is the hereditary algebra of Euclidean type $\mathbb{D}_{5}$ given by the vertices $20,21, \ldots, 24$. The modified algebras (listed in order of creation) are as follows: $B_{1}$ is given by the quiver with vertices $1,2, \ldots, 8$ bound by $\alpha \beta=0$ (we applied (ad $1^{*}$ ) to $C_{1}$ ), $B_{2}$ is given by the quiver with vertices $1,2, \ldots, 11$ bound by $\alpha \beta=0$ (we applied $\left(\operatorname{ad} 1^{*}\right)$ to $\left.B_{1}\right), B_{3}$ is given by the quiver with vertices $1,2, \ldots, 15$ bound by $\alpha \beta=0, \gamma \delta=0$ (we applied (ad $1^{*}$ ) to $\left.B_{2}\right), B_{4}$ is given by the quiver with vertices $1,2, \ldots, 16$ bound by $\alpha \beta=0, \gamma \delta=0, \eta \varepsilon=0$ (we applied ( $\left(\mathrm{dd} 1^{*}\right.$ ) to $\left.B_{3}\right), B_{5}$ is given by the quiver with vertices $20,21, \ldots, 28$ bound by $\varphi_{3} \psi, \varphi_{4} \psi$ (we applied $\left(\operatorname{ad} 1^{*}\right)$ to $\left.C_{2}\right), B_{6}$ is given by the quiver with vertices $20,21, \ldots, 31$ bound by $\varphi_{3} \psi, \varphi_{4} \psi, \xi_{3} \omega_{1}$ (we applied ( $\operatorname{ad} 1^{*}$ ) to $B_{5}$ ), $B_{7}$ is given by the quiver with vertices $20,21, \ldots, 32$ bound by $\varphi_{3} \psi, \varphi_{4} \psi, \xi_{3} \omega_{1}, \zeta_{1} \varphi_{1}, \zeta_{1} \varphi_{2}, \zeta_{2} \xi_{3} \xi_{2} \xi_{1}-\zeta_{1} \psi($ we applied (ad 2) to $\left.B_{6}\right), B_{8}$ is given by the quiver with vertices $20,21, \ldots, 33$ bound by $\varphi_{3} \psi, \varphi_{4} \psi, \xi_{3} \omega_{1}, \zeta_{1} \varphi_{1}$, $\zeta_{1} \varphi_{2}, \zeta_{2} \xi_{3} \xi_{2} \xi_{1}-\zeta_{1} \psi, \pi_{1} \xi_{2}, \pi_{1} \omega_{1}-\pi_{2} \omega_{2}$ (we applied (ad 3) to $B_{7}$ ), $B_{9}=A$ (we applied (ad 5) to $\left.B_{4} \times B_{8}\right)$.

Then the left quasitilted algebra $A^{(l)}$ of $A$ is the convex subcategory of $A$ being the product $A^{(l)}=A_{1}^{(l)} \times A_{2}^{(l)}$, where $A_{1}^{(l)}=k Q_{1}^{(l)} / I_{1}^{(l)}$ is the branch coextension of the tame concealed algebra $C_{1}, Q_{1}^{(l)}$ is a full subquiver of $Q$ given by the vertices $1,2, \ldots, 16$ and $I_{1}^{(l)}=k Q_{1}^{(l)} \cap I$ is the ideal in $k Q_{1}^{(l)}, A_{2}^{(l)}=k Q_{2}^{(l)} / I_{2}^{(l)}$ is the branch coextension of the tame concealed algebra $C_{2}, Q_{2}^{(l)}$ is a full subquiver of $Q$ given by the vertices $20,21, \ldots, 31$ and $I_{2}^{(l)}=k Q_{2}^{(l)} \cap I$ is the ideal in $k Q_{2}^{(l)}$. The right quasitilted algebra $A^{(r)}$ of $A$ is the convex subcategory of $A$ being the product $A^{(r)}=A_{1}^{(r)} \times A_{2}^{(r)}$, where $A_{1}^{(r)}=C_{1}, A_{2}^{(r)}=k Q_{2}^{(r)} / I_{2}^{(r)}$ is the branch extension of the tame concealed algebra $C_{2}, Q_{2}^{(r)}$ is a full subquiver of $Q$ given by the vertices $14,15, \ldots, 24,26,27,28,30,31,32,33$ and $I_{2}^{(r)}=k Q_{2}^{(r)} \cap I$ is the ideal in $k Q_{2}^{(r)}$. 
Note that a hereditary algebra of type $\widetilde{\mathbb{D}}_{6}\left(A_{1}^{(r)}\right)$ is strongly simply connected as well as considered above: branch coextension of $\widetilde{\mathbb{D}}_{6}\left(A_{1}^{(l)}\right)$, branch extension and branch coextension of hereditary algebra of type $\widetilde{\mathbb{D}}_{5}$ (respectively, $A_{2}^{(r)}$ and $A_{2}^{(l)}$ ). Moreover, it follows from [27, Theorem A] and the above construction that $A$ is tame and $\Gamma_{A}$ admits a separating family of almost cyclic coherent components without exceptional configurations of modules. Then, by Theorem 1.2, the algebra $A$ is strongly simply connected.

\section{Further applications}

As an application of Theorem 1.1 and results of $[1,3,4,24,38]$ we shall describe the strongly simply connected tame iterated generalized multicoil enlargements of a tame concealed algebra.

\subsection{Fundamental properties}

Recall that if $A$ is a domestic tubular extension of the tame concealed algebra, then its module category may be described as follows: $\bmod A=\mathcal{P}^{A} \cup \mathcal{T}^{A} \cup \mathcal{Q}^{A}$, where $\mathcal{P}^{A}$ is a postprojective component, $\mathcal{Q}^{A}$ is a preinjective component and $\mathcal{T}^{A}$ is a tubular $\mathbb{P}_{1}(k)$-family separating $\mathcal{P}^{A}$ from $\mathcal{Q}$ (see [34, (4.9)]).

If $A$ is a tubular algebra, then we know from [34, (5.2)] that $A$ is non-domestic of polynomial growth and

$$
\text { ind } A=\mathcal{P}_{0}^{A} \cup \mathcal{T}_{0}^{A} \cup \underset{\gamma \in \mathbb{Q}^{+}}{ } \mathcal{T}_{\gamma}^{A} \cup \mathcal{T}_{\infty}^{A} \cup \mathcal{Q}_{\infty}^{A},
$$

where $\mathcal{P}_{0}^{A}$ is a semi-regular postprojective component, $\mathcal{Q}_{\infty}^{A}$ is a semi-regular preinjective component, $\mathcal{T}_{0}^{A}$ is a $\mathbb{P}_{1}(k)$-family of ray tubes separating $\mathcal{P}_{0}^{A}$ from $\bigcup_{\gamma \in \mathbb{Q}^{+}} \mathcal{T}_{\gamma}^{A} \cup \mathcal{T}_{\infty}^{A} \cup$ $\mathcal{Q}_{\infty}^{A}, \mathcal{T}_{\infty}^{A}$ is a $\mathbb{P}_{1}(k)$-family of coray tubes separating $\mathcal{P}_{0}^{A} \cup \mathcal{T}_{0}^{A} \cup \cup_{\gamma \in \mathbb{Q}^{+}} \mathcal{T}_{\gamma}^{A}$ from $\mathcal{Q}_{\infty}^{A}$ (because $A$ is also a cotubular algebra), and each $\mathcal{T}_{\gamma}^{A}, \gamma \in \mathbb{Q}_{+}$, where $\mathbb{Q}_{+}$is the set of all positive rationals, is a $\mathbb{P}_{1}(k)$-family of stable tubes separating $\mathcal{P}_{0}^{A} \cup \mathcal{T}_{0}^{A} \cup \bigcup_{\delta<\gamma} \mathcal{T}_{\delta}^{A}$ from $\bigcup_{\gamma<\delta} \mathcal{T}_{\delta}^{A} \cup \mathcal{T}_{\infty}^{A} \cup \mathcal{Q}_{\infty}^{A}$.

Remark 9.2 In [24, Section 6] we introduced tame iterated generalized coil enlargements of a tame concealed algebra using admissible operations of types ( $\operatorname{ad} 1),(\operatorname{ad} 2),(\operatorname{ad} 3)$ and (ad 4) for $r=0$. In the case of (ad 4), $r \geq 1$, we add $r$ new projective modules, but in a strictly defined and controlled way (see definition of (ad 4)). Since in the definition of admissible operation ( $\mathrm{ad} 5)$ we use the finite versions (fad 1), (fad 2), (fad 3), (fad 4) of the admissible operations $(\operatorname{ad} 1),(\operatorname{ad} 2),(\operatorname{ad} 3),(\operatorname{ad} 4)$ and the admissible operation $(\operatorname{ad} 4)$, this construction can be easily generalized, as we do below.

\subsection{Iterated generalized multicoil algebras}

Domestic tubular extensions and coextensions and tubular algebras are obtained from a tame concealed algebra by performing a sequence of admissible operations (ad 1$)$ or $\left(\mathrm{ad} 1^{*}\right)$ in the stable tubes of its separating tubular family. We call these algebras 0 -tame iterated generalized multicoil enlargements. 
Let $B_{0}$ be a branch coextension of a (not necessarily connected) tame concealed algebra $C_{0}$, and assume that $B_{0}$ is domestic or tubular (each connected algebra of $B_{0}$ is domestic or tubular). Then ind $B_{0}=\mathcal{P}^{B_{0}} \cup \mathcal{T}^{B_{0}} \cup \mathcal{Q}^{B_{0}}$, where $\mathcal{Q}^{B_{0}}$ is the preinjective component of $\Gamma_{B_{0}}$, and $\mathcal{T}^{B_{0}}$ is a separating tubular family containing injectives. Applying admissible operations (ad 1)-(ad 5) we insert projectives in the coinserted and stable tubes of $\mathcal{T}^{B_{0}}$. We obtain a generalized multicoil enlargement $A_{1}$ of $C_{0}$ with $A_{1}^{(l)}=B_{0}$. If the branch extension $B_{1}=A_{1}^{(r)}$ of $C_{0}$ is tame, we call $\Lambda_{1}=A_{1}$ a l-tame iterated generalized multicoil enlargement.

If $B_{1}$ is domestic, the iteration process stops. If $B_{1}$ is a tubular algebra, then it is a branch coextension of a tame concealed algebra $C_{1}$, and we can write ind $\Lambda_{1}=\mathcal{P}^{\Lambda_{1}} \cup$ $\mathcal{T}^{\Lambda_{1}} \cup \mathcal{Q}^{\Lambda_{1}}$, where $\mathcal{Q}^{\Lambda_{1}}$ is the preinjective component of $\Gamma_{\Lambda_{1}}$, and $\mathcal{T}^{\Lambda_{1}}$ is a separating tubular family containing injectives. More precisely, $\mathcal{T}^{\Lambda_{1}}$ and $\mathcal{Q}^{\Lambda_{1}}$ are, respectively, the last separating tubular family and the preinjective component of $\Gamma_{B_{1}}$ (see [24, Section 6]). Applying admissible operations (ad 1)-(ad 5) we insert projectives in the coinserted and stable tubes of $\mathcal{T}^{\Lambda_{1}}$. We obtain a generalized multicoil enlargement $A_{2}$ of $C_{1}$ with $A_{2}^{(l)}=$ $A_{1}^{(r)}=B_{1}$. If the branch extension $B_{2}=A_{2}^{(r)}$ of $C_{1}$ is tame, we call $\Lambda_{2}$ obtained from $\Lambda_{1}=A_{1}$ by inserting projectives in the tubes of $\mathcal{T}^{\Lambda_{1}}$ a 2-tame iterated generalized multicoil enlargement.

Again, if $B_{2}$ is domestic, the iteration process stops, and if $B_{2}$ is a tubular algebra we are able to iterate the process one more. Inductively, assume that $\Lambda_{n-1}$ is an $(n-1)$-tame iterated generalized multicoil enlargement such that we are able to iterate the process one more. Therefore, there exists a generalized multicoil enlargement $A_{n-1}$ of a tame concealed algebra $C_{n-2}$ such that the branch extension $B_{n-1}=A_{n-1}^{(r)}$ of $C_{n-2}$ is a tubular algebra. Hence $B_{n-1}$ is a branch coextension of a tame concealed algebra $C_{n-1}$, and we can write ind $\Lambda_{n-1}=\mathcal{P}^{\Lambda_{n-1}} \cup \mathcal{T}^{\Lambda_{n-1}} \cup \mathcal{Q}^{\Lambda_{n-1}}$, where $\mathcal{Q}^{\Lambda_{n-1}}$ is the preinjective component of $\Gamma_{\Lambda_{n-1}}$, and $\mathcal{T}^{\Lambda_{n-1}}$ is a separating tubular family containing injectives. Applying admissible operations (ad 1)-(ad 5) we insert projectives in the coinserted and stable tubes of $\mathcal{T}^{\Lambda_{n-1}}$. We obtain a generalized multicoil enlargement $A_{n}$ of $C_{n-1}$ with $A_{n}^{(l)}=A_{n-1}^{(r)}=B_{n-1}$. If the branch extension $B_{n}=A_{n}^{(r)}$ of $C_{n-1}$ is tame, we call $\Lambda_{n}$ obtained from $\Lambda_{n-1}$ by inserting projectives in the tubes of $\mathcal{T}^{\Lambda_{n-1}}$ an $n$-tame iterated generalized multicoil enlargement.

We mention that, by [24, Proposition 6.3] and Remark 9.2, the $n$-tame iterated generalized multicoil enlargement are of polynomial growth. We will call them simply $n$-iterated generalized multicoil algebras.

Example 9.4 In this example, $\Lambda_{n}$, where $n \in\{0,1,2,3\}$, is an $n$-iterated generalized multicoil algebra. $\Lambda_{0}$ is given by the quivers
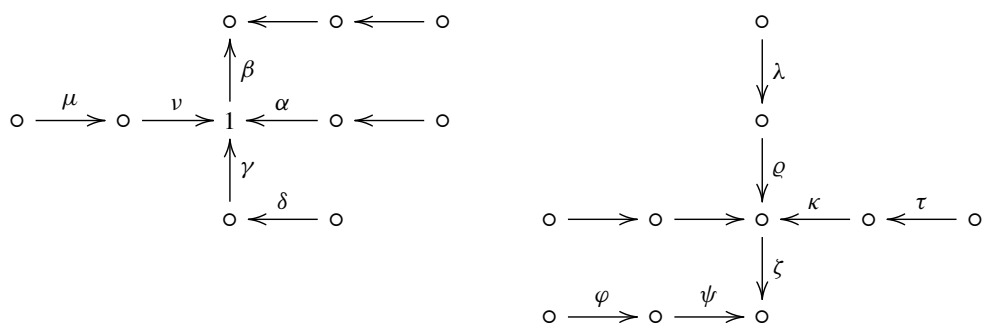
bound by $\alpha \beta=0, \delta \gamma \beta=0, \kappa \zeta=0, \lambda \varrho \zeta=0 ; \Lambda_{1}$ is given by the quiver

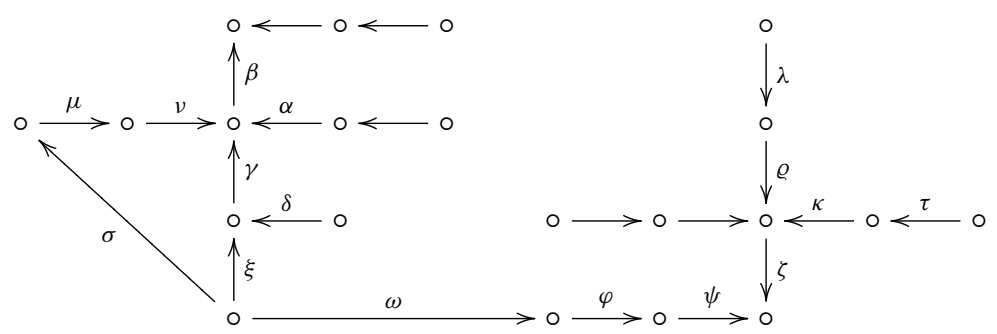

bound by $\alpha \beta=0, \delta \gamma \beta=0, \kappa \zeta=0, \lambda \varrho \zeta=0, \xi \gamma-\sigma \mu \nu=0, \omega \varphi \psi=0 ; \Lambda_{2}$ is given by the quiver

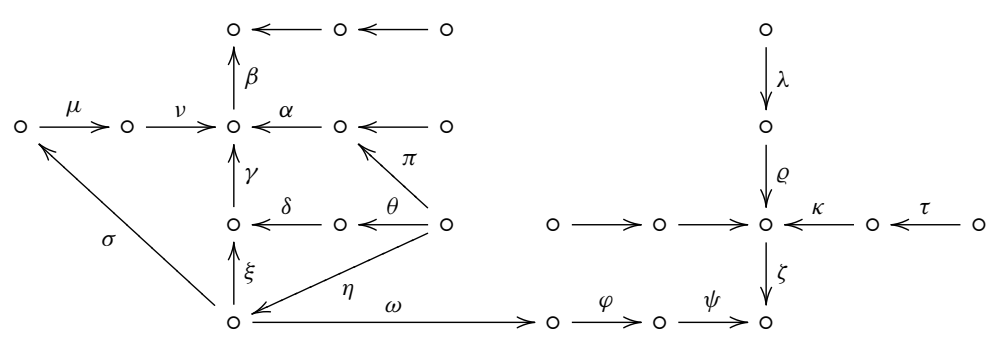

bound by $\alpha \beta=0, \delta \gamma \beta=0, \kappa \zeta=0, \lambda \varrho \zeta=0, \xi \gamma-\sigma \mu \nu=0, \omega \varphi \psi=0, \pi \alpha-\theta \delta \gamma=0$, $\theta \delta-\eta \xi=0, \eta \omega=0 ; \Lambda_{3}$ is given by the quiver

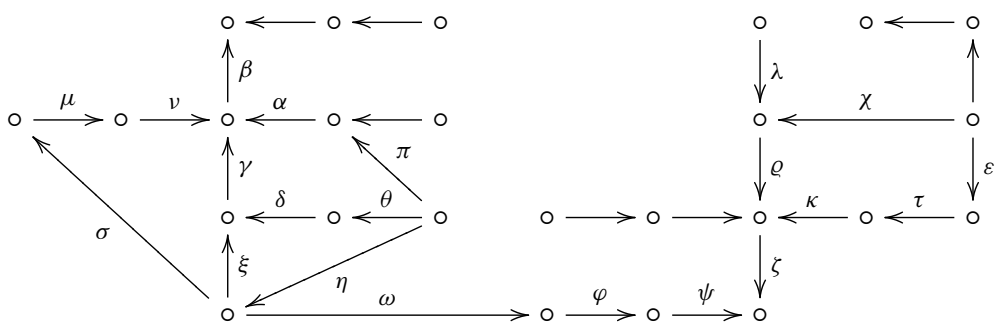

bound by $\alpha \beta=0, \delta \gamma \beta=0, \kappa \zeta=0, \lambda \varrho \zeta=0, \xi \gamma-\sigma \mu \nu=0, \omega \varphi \psi=0, \pi \alpha-\theta \delta \gamma=0$, $\theta \delta-\eta \xi=0, \eta \omega=0, \varepsilon \tau \kappa-\chi \varrho=0$.

Theorem 9.5 Let $A$ be an $n$-iterated generalized multicoil algebra such that $\Gamma_{A}$ does not contain exceptional configurations of modules. Then the following statements are equivalent:

(i) A is strongly simply connected.

(ii) A does not contain a full convex subcategory which is hereditary of type $\widetilde{\mathbb{A}}_{m}$.

(iii) For each $i \in\{1, \ldots, n\}, B_{i}$ does not contain a full convex subcategory which is hereditary of type $\widetilde{\mathbb{A}}_{m}$.

(iv) For each $i \in\{1, \ldots, n\}, C_{i}$ is not hereditary of type $\widetilde{\mathbb{A}}_{m}$.

(v) For each $i \in\{1, \ldots, n\}$, the orbit graph of each directed component of $\Gamma_{B_{i}}$ is a tree.

(vi) For each $i \in\{1, \ldots, n\}, B_{i}$ is strongly simply connected.

(vii) For each $i \in\{1, \ldots, n\}, B_{i}$ satisfies the separation and coseparation conditions. 
Proof Clearly, (i) implies (ii), and (ii) implies (iii). Since the algebras $B_{0}$ and $B_{n}$ are, respectively, either cotilted and tilted of Euclidean type or tubular algebras, and the remaining $B_{i}$ are tubular algebras, the equivalence of (iii), (iv), (v) and (vi) follows from [3, Corollary 2.3] and [1, Theorem 1.6]. By [38, Theorem 4.1], (vi) implies (vii). If the algebra $B_{i}$ is tubular, then by [1, Theorem 1.6], (vii) implies (iii), and if $B_{i}$ is domestic, then by [3, Corollary 2.3] and [1, Lemma 1.5], (vii) implies (iii). It remains to show that (vi) implies (i). We proceed by induction on $n$. If $n=1$, the statement follows from Theorem 1.1. Assume that $n>1$, and that the statement holds for any $k \leq n-1$. If $A=\Lambda_{n}$ is not strongly simply connected, then by Theorem 2.6, it contains an irreducible cycle $w$ which is not a contour, or an irreducible contour $w$ which is not naturally contractible. By induction, $w$ must contain a source lying in $B_{n}$ but not in $C_{n-1}$, and a sink lying in $B_{0}$ but not in $C_{0}$. As in the proof of Theorem 1.1, we may replace $w$ by an irreducible cycle $w^{\prime}$ which is not a contour, but lies in $\Lambda_{n-1}$, a contradiction to the induction hypothesis.

\section{Concluding remarks}

As a direct consequence of Theorem 1.2, Corollary 1.3 and results of [38] we obtain the following corollaries.

Corollary 10.1 Let A be a tame algebra with a separating family of almost cyclic coherent components in $\Gamma_{A}$ without exceptional configurations of modules. Then the following statements are equivalent:

(i) A is strongly simply connected.

(ii) A is simply connected and does not contain a full convex subcategory which is hereditary of type $\widetilde{\mathbb{A}}_{n}$.

(iii) $H^{1}(\underset{\sim}{A})=0$ and $A$ does not contain a full convex subcategory which is hereditary of type $\widetilde{\mathbb{A}}_{n}$.

Corollary 10.2 Let A be a cycle-finite algebra with a separating family of almost cyclic coherent components in $\Gamma_{A}$ without exceptional configurations of modules. The following statements are equivalent:

(i) A is strongly simply connected.

(ii) A is simply connected and does not contain a full convex subcategory which is hereditary of type $\widetilde{\mathbb{A}}_{n}$.

(iii) $H^{1}(\underset{\widetilde{A}}{A}=0$ and $A$ does not contain a full convex subcategory which is hereditary of type $\widetilde{\mathbb{A}}_{n}$.

Open Access This article is licensed under a Creative Commons Attribution 4.0 International License, which permits use, sharing, adaptation, distribution and reproduction in any medium or format, as long as you give appropriate credit to the original author(s) and the source, provide a link to the Creative Commons licence, and indicate if changes were made. The images or other third party material in this article are included in the article's Creative Commons licence, unless indicated otherwise in a credit line to the material. If material is not included in the article's Creative Commons licence and your intended use is not permitted by statutory regulation or exceeds the permitted use, you will need to obtain permission directly from the copyright holder. To view a copy of this licence, visit http://creativecommonshorg/licenses/by/4.0/. 


\section{References}

1. Assem, I.: Strongly simply connected derived tubular algebras. In: Representations of Algebras, Lecture Notes in Pure and Appl. Math, vol. 224, pp. 21-29. Dekker, New York (2002)

2. Assem, I., Coelho, F.U., Trepode, S.: Simply connected tame quasi-tilted algebras. J. Pure Appl. Algebra 172, 139-160 (2002)

3. Assem, I., Liu, S.: Strongly simply connected tilted algebras. Ann. Sci. Math. Québec 21, 13-22 (1997)

4. Assem, I., Liu, S.: Strongly simply connected algebras. J. Algebra 207, 449-477 (1998)

5. Assem, I., Liu, S., de la Peña, J.A.: The strong simple connectedness of a tame tilted algebra. Comm. Algebra 28, 1553-1565 (2000)

6. Assem, I., de la Peña, J.A.: The fundamental groups of a triangular algebra. Comm. Algebra 24, 187-208 (1996)

7. Assem, I., Simson, D., Skowroński, A.: Elements of the representation theory of associative algebras 1: Techniques of representation theory london mathematical society student texts, vol. 65 . Cambridge University Press, Cambridge (2006)

8. Assem, I., Skowroński, A.: On some classes of simply connected algebras. Proc. London Math. Soc. 56, 417-450 (1988)

9. Assem, I., Skowroński, A.: Minimal representation-infinite coil algebras. Manuscr. Math. 67, 305-331 (1990)

10. Assem, I., Skowroński, A.: Multicoil algebras. In: Representations of Algebras. CMS Conf. Proc. 14, Amer. Math. Soc., Providence, RI, pp. 29-68 (1993)

11. Assem, I., Skowroński, A., Tomé, B.: Coil enlargements of algebras. Tsukuba J. Math. 19, 453-479 (1995)

12. Bautista, R., Larrion, F., Salmeron, L.: On simply connected algebras. J. London. Math. Soc. 27, 212-220 (1983)

13. Bongartz, K., Gabriel, P.: Covering spaces in representation theory. Invent. Math. 65, 331-378 (1982)

14. Coelho, F.U., Martins, M.I.R., Tomé, B.: Strongly simply connected coil algebras. Colloq. Math. 99, 91-110 (2004)

15. Crawley-Boevey, W.: On tame algebras and bocses. Proc. London Math. Soc. 56, 451-483 (1988)

16. Drozd, Y.A.: Tame and wild matrix problems. In: Representation Theory II, Lecture Notes in Math. 832, pp. 242-258. Springer (1980)

17. Gerstenhaber, M.: On the deformation of rings and algebras. Ann. of Math. 79, 59-103 (1964)

18. Happel, D., Vossieck, D.: Minimal algebras of infinite representation type with preprojective component. Manuscripta Math. 42, 221-243 (1983)

19. Hoshino, M.: Modules without self-extensions and Nakayama's conjecture. Archiv. Math. (Basel) 43, 493-500 (1984)

20. Kerner, O.: Tilting wild algebras. J. London Math. Soc. 39, 29-47 (1989)

21. Lenzing, H., Meltzer, H.: Tilting sheaves and concealed-canonical algebras. In: Representation Theory of Algebras, CMS Conference Proc., vol. 18, pp. 455-473 (1996)

22. Lenzing, H., de la Peña, J.A.: Concealed-canonical algebras and separating tubular families. Proc. London Math. Soc. 78, 513-540 (1999)

23. Lenzing, H., Skowroński, A.: Quasi-tilted algebras of canonical type. Colloq. Math. 71, 161-181 (1996)

24. Malicki, P.: Generalized coil enlargements of algebras. Colloq. Math. 76, 57-83 (1998)

25. Malicki, P., de la Peña, J.A., Skowroński, A.: Cycle-finite module categories. In: Algebras, Quivers and Representations - Abel Symposium 2011. Abel Symposia 8, pp. 209-252. Springer (2013)

26. Malicki, P., Skowroński, A.: Almost cyclic coherent components of an Auslander-Reiten quiver. J. Algebra 229, 695-749 (2000)

27. Malicki, P., Skowroński, A.: Algebras with separating almost cyclic coherent Auslander-Reiten components. J. Algebra 291, 208-237 (2005)

28. Malicki, P., Skowroński, A.: On the additive categories of generalized standard almost cyclic coherent Auslander-Reiten components. J. Algebra 316, 133-146 (2007)

29. Malicki, P., Skowroński, A.: Algebras with separating Auslander-Reiten components. In: Representations of Algebras and Related Topics, European Math. Soc. Series Congress Reports, European Math. Soc. Publ. House, Zürich, pp. 251-353 (2011)

30. Malicki, P., Skowroński, A.: Hochschild cohomology of generalized multicoil algebras. Colloq. Math. 136, 231-254 (2014) 
31. Malicki, P., Skowroński, A.: The structure and homological properties of generalized standard Auslander-Reiten components. J. Algebra 518, 1-39 (2019)

32. Reiten, I., Skowroński, A.: Characterizations of algebras with small homological dimensions. Adv. Math. 179, 122-154 (2003)

33. Ringel, C.M.: Separating tubular series. In: Séminare d'Algébre Paul Dubreil et Marie-Paul Malliavin, Lecture Notes in Math., vol. 1029, pp. 134-158. Springer, Berlin (1983)

34. Ringel, C.M.: Tame Algebras and Integral Quadratic Forms, p. 1984. Springer, Berlin (1099). Lecture Notes in Math.

35. Simson, D., Skowroński, A.: Elements of the Representation Theory of Associative Algebras 2: Tubes and Concealed Algebras of Euclidean Type London Mathematical Society Student Texts, vol. 71. Cambridge University Press, Cambridge (2007)

36. Simson, D., Skowroński, A.: Elements of the Representation Theory of Associative Algebras 3 : Representation-Infinite Tilted Algebras London Mathematical Society Student Texts, vol. 72. Cambridge University Press, Cambridge (2007)

37. Skowroński, A.: Algebras of polynomial growth. In: Topics in Algebra, vol. 26, pp. 535-568 (1990). Part 1, PWN Warsaw

38. Skowroński, A.: Simply connected algebras and Hochschild cohomologies. In: Representations of Algebras, CMS Conference Proc., vol. 14, pp. 431-447 (1993)

39. Skowroński, A.: Generalized standard Auslander-Reiten components. J. Math. Soc. Japan 46, 517-543 (1994)

40. Skowroński, A.: Cycle-finite algebras. J. Pure Appl. Algebra 103, 105-116 (1995)

Publisher's Note Springer Nature remains neutral with regard to jurisdictional claims in published maps and institutional affiliations. 